\title{
Elliptic multiple zeta values and one-loop superstring amplitudes
}

\author{
Johannes Broedel, ${ }^{a}$ Carlos R. Mafra, ${ }^{b}$ Nils Matthes $^{c}$ and Oliver Schlotterer ${ }^{d}$ \\ ${ }^{a}$ Institut für Theoretische Physik, Eidgenössische Technische Hochschule Zürich, \\ Wolfgang-Pauli-Strasse 27, 8093 Zürich, Switzerland \\ ${ }^{b}$ Department of Applied Mathematics and Theoretical Physics, University of Cambridge, \\ Wilberforce Road, Cambridge CB3 OWA, U.K. \\ ${ }^{c}$ Fachbereich Mathematik, Universität Hamburg, \\ Bundesstraße 55, 20146 Hamburg, Germany \\ ${ }^{d}$ Max-Planck-Institut für Gravitationsphysik, Albert-Einstein-Institut, \\ Am Mühlenberg 1, 14476 Potsdam, Germany \\ E-mail: jbroedel@ethz.ch, c.r.mafra@damtp.cam.ac.uk, \\ nils.matthes@uni-hamburg.de, olivers@aei.mpg.de
}

ABStRact: We investigate iterated integrals on an elliptic curve, which are a natural genus-one generalization of multiple polylogarithms. These iterated integrals coincide with the multiple elliptic polylogarithms introduced by Brown and Levin when constrained to the real line. At unit argument they reduce to an elliptic analogue of multiple zeta values, whose network of relations we start to explore. A simple and natural application of this framework are one-loop scattering amplitudes in open superstring theory. In particular, elliptic multiple zeta values are a suitable language to express their low energy limit. Similar to the techniques available at tree-level, our formalism allows to completely automatize the calculation.

Keywords: Scattering Amplitudes, Superstrings and Heterotic Strings, Conformal Field Models in String Theory

ARXIV EPRINT: 1412.5535 


\section{Contents}

1 Introduction 1

2 Iterated integrals on an elliptic curve $\quad 3$

2.1 Multiple polylogarithms 3

2.1.1 Removing the argument $z$ from the label 4

2.2 Iterated integrals on an elliptic curve 5

$\begin{array}{lll}2.2 .1 & \text { Elliptic multiple zeta values } & 7\end{array}$

2.2.2 Removing the argument $z$ from the label 8

$\begin{array}{lll}2.2 .3 & \text { Relations among elliptic multiple zeta values } & 12\end{array}$

3 The functions $f^{(n)}$ on the elliptic curve 13

$\begin{array}{lll}3.1 & \text { Motivation } & 13\end{array}$

$\begin{array}{lll}3.2 & \text { Doubly-periodic functions and generating series } & 14\end{array}$

$\begin{array}{lll}3.2 .1 & \text { Some doubly-periodic functions } & 15\end{array}$

$\begin{array}{lll}3.2 .2 & \text { The Eisenstein-Kronecker series } & 15\end{array}$

$\begin{array}{ll}3.2 .3 & \text { Restoring double periodicity and modularity } \\ & 16\end{array}$

3.3 Definition and properties of the weighting functions $f^{(n)} \quad 16$

$\begin{array}{ll}\text { 3.3.1 Definition of } f^{(n)} & 16\end{array}$

$\begin{array}{ll}\text { 3.3.2 Properties of } f^{(n)} & 17\end{array}$

$\begin{array}{lll}3.3 .3 & q \text {-expansions of } f^{(n)} & 18\end{array}$

4 The one-loop four-point amplitude in open string theory 19

$\begin{array}{ll}4.1 \text { The four-point amplitude } & 19\end{array}$

$\begin{array}{ll}4.2 \text { The } \alpha^{\prime} \text {-expansion } & 21\end{array}$

$\begin{array}{lll}4.3 & \text { Elliptic multiple zeta values } & 22\end{array}$

4.3.1 First order in $P_{i j}$ : integrals $c_{i}^{1} \quad 23$

4.3.2 Second order in $P_{i j}$ : integrals $c_{i}^{2} \quad 23$

4.3.3 Integration techniques for $P_{23}, P_{24}, P_{34}$

4.3.4 Third order in $P_{i j}$ : integrals $c_{i}^{3} \quad 24$

$\begin{array}{lll}\text { 4.3.5 Assembling the results } & 25\end{array}$

4.4 On the $q$-expansion of eMZVs and the string amplitude 25

4.4.1 The simplest $q$-expansions 25

4.4.2 The constant piece of eMZVs and the $\alpha^{\prime}$-derivative 26

5 Multi-particle one-loop string amplitudes and $f^{(n)} \quad \mathbf{2 7}$

$\begin{array}{ll}\text { 5.1 The five-point open string amplitude } & 27\end{array}$

5.2 Functions $f^{(n)}$ from the RNS formalism 28

$\begin{array}{lll}\text { 5.2.1 Parity-even RNS amplitudes } & 28\end{array}$

5.2.2 Parity-odd RNS amplitudes 31

6 Discussion and further directions $\quad 31$ 
B Identities for iterated integrals $\quad 33$

B.1 Total derivatives 33

B.2 Recursive removal of the argument from the labels 34

B.3 Eliminating labels $a_{j}=z$ at length three $\quad 35$

C Trigonometric integrals $\quad 35$

D Cycle index of the symmetric group and the $f^{(n)}$ functions 36

\section{Introduction}

In recent years, we have witnessed numerous fruitful interactions between number theory and particle physics. A particularly rich domain of intersection are iterated integrals, which prominently appear in scattering amplitudes in field theories and string theories. For a large class of Feynman and worldsheet integrals, multiple polylogarithms were recognized as a suitable language to cast results into a manageable form, see e.g. refs. [1-4]. In a variety of cases, the polylogarithms' Hopf algebra structure [5-8] paved the way towards efficient manipulations and the recognition of the simplicity hidden in the resulting scattering amplitudes.

However, a growing list of iterated integrals from various field and string theories implies that multiple polylogarithms do not mark the end of the rope in terms of transcendental functions appearing in scattering amplitudes. For example, multiple polylogarithms fail to capture the two-loop sunset integral with non-zero masses [9-11], an eight-loop graph in $\phi^{4}$ theory $[12,13]$ as well as the ten-point two-loop $\mathrm{N}^{3} \mathrm{MHV}$ amplitude in $\mathcal{N}=4$ superYang-Mills (sYM) theory [14]. The sunset integral and its generalization have recently been expressed in terms of elliptic di- and trilogarithms [10, 11, 15], whose connection to the language suggested below remains to be worked out. Considering in addition their appearance in one-loop open-string amplitudes, the situation calls for a systematic study and classification of the entire family of elliptic iterated integrals. ${ }^{1}$

In the present article, we propose a framework for elliptic iterated integrals (or eIIs for short) and the associated periods, elliptic multiple zeta values (eMZVs). The framework aims at expressing scattering amplitudes in a variety of theories, and we here apply the techniques to one-loop amplitudes in open string theory as a first example. The language employed in the present article is primarily inspired by refs. [16, 17], while refs. [18-22] contain further information on the mathematical background.

As opposed to multiple polylogarithms, which can be defined using just one type of differential form, elliptic iterated integrals require an infinite tower thereof [16]. These

\footnotetext{
${ }^{1}$ The elliptic iterated integrals discussed in this work shall not be confused with elliptic integrals determining the arc length of an ellipse.
} 
differential forms are based on a certain non-holomorphic extension of a classical EisensteinKronecker series [16, 23], and we show how they can be used to naturally characterize and label elliptic iterated integrals as well as eMZVs. We investigate their relations, which results in constructive algorithms to perform amplitude computations.

In the same way as multiple zeta values (MZVs) arise from multiple polylogarithms at unit argument, the evaluation of iterated integrals along a certain path of an elliptic curve leads to structurally interesting periods, the eMZVs [17] mentioned above. These are certain analogues of the standard MZVs, which are related to elliptic associators [24] in the same way as MZVs are related to the Drinfeld associator [25-27]. However, the precise connection is beyond the scope of the current article. Given their ubiquitous appearance in the subsequent string amplitude computation, we will investigate eMZVs and discuss some of their properties as well as their $\mathbb{Q}$-linear relations.

The description of string scattering amplitudes via punctured Riemann surfaces at various genera directly leads to iterated integrals at the corresponding loop order. In particular, the disk integrals in open-string ${ }^{2}$ tree-level amplitudes closely resemble multiple polylogarithms. Initially addressed via hypergeometric functions in refs. [34, 35], the $\alpha^{\prime}$-expansion of disk amplitudes finally proved to be a rich laboratory for MZVs. Their pattern of appearance has been understood in terms of mathematical structures such as motivic MZVs [7, 29] and the Drinfeld associator [36-38]. Explicit expressions with any number of open-string states can be determined using polylogarithm manipulations [3] or a matrix representation of the associator [38]. A variety of examples are available for download at the website [39].

The calculation of one-loop open-string amplitudes involves worldsheet integrals of cylinder and Möbius-strip topology [40]. In the current article, we focus on iterated integrals over a single cylinder boundary and leave the other topologies for later. Recognizing the cylinder as a genus-one surface with boundaries, it is not surprising that the $\alpha^{\prime}$-expansion of one-loop open-string amplitudes is a natural, simple and representative framework for the application of eIIs and eMZVs. We will explicitly perform calculations at four and five points for low orders in $\alpha^{\prime}$ in order to demonstrate their usefulness. Higher multiplicities and orders in $\alpha^{\prime}$ are argued to yield eMZVs and Eisenstein series on general grounds. In summary, one-loop string amplitudes turn out to be an ideal testing ground for the study of eMZVs, in particular because they appear in a more digestible context as compared to their instances in field theory.

This article is organized as follows: in section 2, we start by reviewing multiple polylogarithms and show, how their structure suggests a generalization to genus one. The appropriate differential forms and doubly-periodic functions are discussed and put into a larger mathematical context in section 3. Section 4 is devoted to the application of eIIs and eMZVs to the four-point one-loop amplitude of the open string, while section 5 contains a discussion of its multi-particle generalization.

\footnotetext{
${ }^{2}$ In comparison to open-string amplitudes at tree-level, MZVs occurring in closed-string tree amplitudes [28, 29] are constrained by the single-valued projection, see [30, 31] for mathematics and [32, 33] for physics literature.
} 


\section{Iterated integrals on an elliptic curve}

After recalling the definition of multiple polylogarithms as well as several conventions, we will introduce elliptic iterated integrals (eIIs) as their genus-one analogues. While we will limit ourselves to basic definitions and calculational tools in the current section, a thorough introduction to the mathematical background of doubly-periodic functions will be provided in section 3 .

\subsection{Multiple polylogarithms}

Multiple polylogarithms are defined by ${ }^{3}$

$$
G\left(a_{1}, a_{2}, \ldots, a_{n} ; z\right) \equiv \int_{0}^{z} \frac{\mathrm{d} t}{t-a_{1}} G\left(a_{2}, \ldots, a_{n} ; t\right)
$$

where $G(; z) \equiv 1$ apart from $G(\vec{a} ; 0)=G(; 0)=0$. Below, we will refer to $\vec{a}=\left(a_{1}, \ldots, a_{n}\right)$ as the label and call $z$ the argument of the polylogarithm $G$. Powers of ordinary logarithms can be conveniently represented in terms of multiple polylogarithms via

$$
\begin{aligned}
& G(\underbrace{0,0, \ldots, 0}_{n} ; z)=\frac{1}{n !} \ln ^{n} z, \quad G(\underbrace{1,1 \ldots, 1}_{n} ; z)=\frac{1}{n !} \ln ^{n}(1-z) \quad \text { and } \\
& G(\underbrace{a, a, \ldots, a}_{n} ; z)=\frac{1}{n !} \ln ^{n}\left(1-\frac{z}{a}\right) .
\end{aligned}
$$

In addition, multiple polylogarithms satisfy the scaling property

$$
G\left(k a_{1}, k a_{2}, \ldots, k a_{n} ; k z\right)=G\left(a_{1}, a_{2}, \ldots, a_{n} ; z\right), \quad k \neq 0, \quad a_{n} \neq 0, \quad z \neq 0
$$

whose interplay with a general shuffle regularization will be discussed below eq. (2.9). Another property is referred to as the Hölder convolution [44]: for $a_{1} \neq 1$ and $a_{n} \neq 0$ one finds

$$
G\left(a_{1}, \ldots, a_{n} ; 1\right)=\sum_{k=0}^{n}(-1)^{k} G\left(1-a_{k}, \ldots, 1-a_{1} ; 1-\frac{1}{p}\right) G\left(a_{k+1}, \ldots, a_{n} ; \frac{1}{p}\right)
$$

for all $p \in \mathbb{C} \backslash\{0\}$. Multiple polylogarithms constitute a graded commutative algebra with the shuffle product $[5-8]$

$$
\begin{aligned}
G\left(a_{1}, \ldots, a_{r} ; z\right) G\left(a_{r+1}, \ldots, a_{r+s} ; z\right) & =\sum_{\sigma \in \Sigma(r, s)} G\left(a_{\sigma(1)}, \ldots, a_{\sigma(r+s)} ; z\right) \\
& \equiv G\left(\left(a_{1}, \ldots, a_{r}\right) \amalg\left(a_{r+1}, \ldots, a_{r+s}\right) ; z\right)
\end{aligned}
$$

where the shuffle $\Sigma(r, s)$ is the subset of the permutation group $S_{r+s}$ acting on $\left\{a_{1}, \ldots, a_{r+s}\right\}$ which leaves the order of the elements of the individual tuples $\left\{a_{1}, \ldots, a_{r}\right\}$ and $\left\{a_{r+1}, \ldots, a_{r+s}\right\}$ unchanged. The unit element for shuffling is $G(; z)=1$.

\footnotetext{
${ }^{3}$ The conventions for multiple polylogarithms used in this paper agree with those in refs. [5, 29, 41]. Other aspects of multiple polylogarithms are discussed for example in references [42, 43].
} 
MZVs are special cases of multiple polylogarithms with labels $a_{i} \in\{0,1\}$ evaluated at argument $z=1$ :

$$
\zeta_{n_{1}, \ldots, n_{r}}=(-1)^{r} G(\underbrace{0,0, \ldots, 0,1}_{n_{r}}, \ldots, \underbrace{0,0, \ldots, 0,1}_{n_{1}} ; 1)
$$

where the numbers below the underbraces denote the number of entries. ${ }^{4}$

From the definition (2.1) it is obvious that multiple polylogarithms diverge when either $a_{1}=z$ or $a_{n}=0$. As discussed in refs. [5,6], the general idea for regularizing the integrals is to slightly move the endpoints of the integration by a small parameter and to afterwards expand in this parameter. The regularized value of the polylogarithm is defined to be the piece independent of the regularization parameter, which can be extracted using shuffle relations. For the case where $a_{1}=z$ the regularized value can be obtained via

$$
\begin{aligned}
G\left(z, a_{2}, \ldots, a_{n} ; z\right)= & G(z ; z) G\left(a_{2}, \ldots, a_{n} ; z\right)-G\left(a_{2}, z, a_{3}, \ldots, a_{n} ; z\right) \\
& -G\left(a_{2}, a_{3}, z, a_{4}, \ldots, a_{n} ; z\right)-\ldots-G\left(a_{2}, \ldots, a_{n}, z ; z\right)
\end{aligned}
$$

where one defines

$$
G(z, \ldots, z ; z)=0 .
$$

The situation, where $a_{n}=0$ can be dealt with accordingly

$$
\begin{gathered}
G\left(a_{1}, a_{2}, \ldots, a_{n-1}, 0 ; z\right)=G\left(a_{1}, a_{2}, \ldots, a_{n-1} ; z\right) G(0 ; z)-G\left(a_{1}, a_{2}, \ldots, 0, a_{n-1} ; z\right) \\
-G\left(a_{1}, a_{2}, \ldots, 0, a_{n-2}, a_{n-1} ; z\right)-\ldots-G\left(0, a_{2}, \ldots, a_{n-1} ; z\right)
\end{gathered}
$$

where now, however, $G(0 ; z)=\ln (z) \neq 0$. Although the above rewriting keeps the pure logarithms explicit, it will nevertheless prove convenient in order to bypass subtleties of the identity eq. (2.11) below. Multiple polylogarithms are understood to be shuffle-regularized in a way compatible with eq. (2.3).

Regularization of multiple polylogarithms can be straightforwardly translated to MZVs. All MZVs $\zeta_{n_{1}, \ldots, n_{r}}$ with $n_{r}=1$ are defined by their shuffled version eq. (2.7). Employing eq. (2.3), one finds $G(1, \ldots, 1 ; 1)=0$ from eq. (2.8) immediately.

\subsubsection{Removing the argument $z$ from the label}

Starting from an arbitrary iterated integral, the corresponding polylogarithm can not always be determined straightforwardly: whenever the argument appears in the label $\vec{a}$, an integration using eq. (2.1) is impossible. Solving this problem requires a rewriting of the multiple polylogarithm

$$
G\left(\left\{0, a_{1}, a_{2}, \ldots, a_{n}, z\right\} ; z\right)
$$

in terms of polylogarithms whose labels are free of the argument. In the above equation $\{a, b, \ldots\}$ refers to a word built from the letters $a, b, \ldots$. Polylogarithms of the special form $G(\vec{a}, z)$ with $a_{i} \in\{0, z\}$ can be rescaled to yield MZVs using eq. (2.3) provided that the last entry of $\vec{a}$ is different from zero. In a generic situation, the relation [3]

$$
G\left(a_{1}, \ldots, a_{i-1}, z, a_{i+1}, \ldots, a_{n} ; z\right)=G\left(a_{i-1}, a_{1}, \ldots, a_{i-1}, \hat{z}, a_{i+1}, \ldots, a_{n} ; z\right)
$$

\footnotetext{
${ }^{4}$ Our convention for MZVs agrees with refs. [5, 29, 45].
} 


$$
\begin{aligned}
& -G\left(a_{i+1}, a_{1}, \ldots, a_{i-1}, \hat{z}, a_{i+1}, \ldots, a_{n} ; z\right) \\
& -\int_{0}^{z} \frac{\mathrm{d} t}{t-a_{i-1}} G\left(a_{1}, \ldots, \hat{a}_{i-1}, t, a_{i+1}, \ldots, a_{n} ; t\right) \\
& +\int_{0}^{z} \frac{\mathrm{d} t}{t-a_{i+1}} G\left(a_{1}, \ldots, a_{i-1}, t, \hat{a}_{i+1}, \ldots, a_{n} ; t\right) \\
& +\int_{0}^{z} \frac{\mathrm{d} t}{t-a_{1}} G\left(a_{2}, \ldots, a_{i-1}, t, a_{i+1}, \ldots, a_{n} ; t\right)
\end{aligned}
$$

allows to recursively remove the argument $z$ from the labels of a multiple polylogarithm, because the expressions on the right-hand side either have shorter labels or are free of $z$. A hat denotes the omission of the respective label, and it is assumed that at least one $a_{j} \neq 0$. The availability of a recursive formula like eq. (2.11) is intrinsic to the moduli space of Riemann spheres with marked points [46]. An explicit discussion including algorithms is ref. [47].

As an identity similar to eq. (2.11) will be crucial in deriving relations for eIIs in subsection 2.2 below, let us briefly comment on the application and generalization of eq. (2.11): if the argument $z$ appears multiple times in the label $\vec{a}$, the first four terms on the right hand side (terms (2.11a) to (2.11d)) have to be evaluated for each occurrence of $z$. The reduction will lead to expressions where the labels of the polylogarithms on the right hand side are independent of $z$ or shorter, which is ensured by cancellations between neighboring terms. If $a_{n}=z$, the term $(2.11 \mathrm{~d})$ has to be dropped and the term (2.11b) needs to be altered to $-G\left(0, a_{1}, \ldots, a_{i-1}, \hat{z} ; z\right)$.

Multiple polylogarithms with $a_{1}=z$ require special attention as well. However, in order to keep the exposition simple, we will assume that those polylogarithms have already been taken care of by applying the shuffle regularization rule eq. (2.7).

The following examples (with $a_{j} \neq z$ ) are typical relations derived from the above identity:

$$
\begin{aligned}
G\left(a_{1}, 0, z ; z\right) & =G\left(0,0, a_{1} ; z\right)-G\left(0, a_{1}, a_{1} ; z\right)-G\left(a_{1} ; z\right) \zeta_{2} \\
G\left(a_{1}, z, a_{2} ; z\right) & =G\left(a_{1}, a_{1}, a_{2} ; z\right)-G\left(a_{2}, 0, a_{1} ; z\right)+G\left(a_{2}, a_{1}, a_{1} ; z\right)-G\left(a_{2}, a_{1}, a_{2} ; z\right) .
\end{aligned}
$$

Proving eq. (2.11) is straightforward. It relies on writing the polylogarithm on the left hand side as the integral over its total derivative and using partial fraction as well as relations (A.1) to (A.3) in appendix A. Finally, let us note that eq. (2.11) preserves shuffle regularization. The complete proof of eq. (2.11) as well as numerous examples are contained in section 5 of ref. [3]. A collection of identities between MZVs can be found in the multiple zeta value data mine [48].

\subsection{Iterated integrals on an elliptic curve}

In this subsection we are going to take a first look at eIIs. In the following exposition, we will omit several mathematical details, which will be discussed in section 3 below. As eIIs will turn out to be a generalization of the multiple polylogarithms discussed above, we will follow the structure of the previous subsection closely.

In eq. (2.1), the differential $\mathrm{d} t$ is weighted by

$$
\frac{1}{t-a_{i}}
$$


which yields iterated integrals on the genus-zero curve $\mathbb{C} \backslash\left\{a_{1}, \ldots, a_{n}\right\}$. Here, we propose a generalization to eIIs. An infinite number of weighting functions $f^{(n)}$ of weights $n=$ $0,1,2, \ldots$ is necessary, whose appearance will be justified and whose precise definition will be provided in section 3. They lead to eIIs in the same way as does eq. (2.13) at genus zero. Accordingly, the functions $f^{(n)}(z, \tau)$ are doubly periodic with respect to the two cycles of the torus, with modular parameter $\tau$ in the upper half plane

$$
f^{(n)}(z, \tau)=f^{(n)}(z+1, \tau) \quad \text { and } \quad f^{(n)}(z, \tau)=f^{(n)}(z+\tau, \tau) .
$$

Below, we are going to suppress the $\tau$-dependence and will simply write $f^{(n)}(z)$. As will be explained in subsection 3.3, the functions $f^{(n)}$ are known for all non-negative integer weights $n$. In particular they are non-holomorphic and expressible in terms of the odd Jacobi function $\theta_{1}(z, \tau)$, e.g.

$$
\begin{aligned}
& f^{(0)}(z) \equiv 1, \quad f^{(1)}(z) \equiv \partial \ln \theta_{1}(z, \tau)+2 \pi i \frac{\operatorname{Im} z}{\operatorname{Im} \tau} \\
& f^{(2)}(z) \equiv \frac{1}{2}\left[\left(\partial \ln \theta_{1}(z, \tau)+2 \pi i \frac{\operatorname{Im} z}{\operatorname{Im} \tau}\right)^{2}+\partial^{2} \ln \theta_{1}(z, \tau)-\frac{1}{3} \frac{\theta_{1}^{\prime \prime \prime}(0, \tau)}{\theta_{1}^{\prime}(0, \tau)}\right]
\end{aligned}
$$

where $\partial$ and ' denote a derivative in the first argument of $\theta_{1}$. Their parity alternates depending on the weight $n$ :

$$
f^{(n)}(-z)=(-1)^{n} f^{(n)}(z) .
$$

The functions $f^{(n)}$ are defined for arbitrary complex arguments $z$. Restricting to real arguments $z$, however, will not only simplify eqs. (2.15) and (2.16) but in addition lead to the system of iterated integrals appropriate for the one-loop open-string calculations in sections 4 and 5 below. Hence, in the remainder of the current section, any argument and label of the eIIs to be defined is assumed to be real. We will comment on the additional ingredients required for generic complex arguments $z$ and relate them to multiple elliptic polylogarithms in subsection 3.1.

Employing the functions $f^{(n)}$, eIIs are defined in analogy to eq. (2.1) via

$$
\Gamma\left(\begin{array}{cccc}
n_{1} & n_{2} & \ldots & n_{r} \\
a_{1} & a_{2} & \ldots & a_{r}
\end{array} ; z\right) \equiv \int_{0}^{z} \mathrm{~d} t f^{\left(n_{1}\right)}\left(t-a_{1}\right) \Gamma\left(\begin{array}{cccc}
n_{2} & \ldots & n_{r} \\
a_{2} & \ldots & a_{r}
\end{array} ; t\right),
$$

where the recursion starts with $\Gamma(; z) \equiv 1$. Following the terminology used for $f^{(n)}$ above, the eII in eq. (2.18) is said to have weight $\sum_{i=1}^{r} n_{i}$, and the number $r$ of integrations will be referred to as its length.

The definition of eIIs directly implies a shuffle relation with respect to the combined letters $A_{i} \equiv{ }_{a_{i}}^{n_{i}}$ describing the integration weights $f^{\left(n_{i}\right)}\left(z-a_{i}\right)$,

$$
\Gamma\left(A_{1}, A_{2}, \ldots, A_{r} ; z\right) \Gamma\left(B_{1}, B_{2}, \ldots, B_{q} ; z\right)=\Gamma\left(\left(A_{1}, A_{2}, \ldots, A_{r}\right) ш\left(B_{1}, B_{2}, \ldots, B_{q}\right) ; z\right),
$$

where the shuffle symbol has been defined in eq. (2.5). Another immediate consequence of definition (2.18) is the reflection identity

$$
\Gamma\left(\begin{array}{cccc}
n_{1} & n_{2} & \ldots & n_{r} \\
a_{1} & a_{2} & \ldots & a_{r}
\end{array} ; z\right)=(-1)^{n_{1}+n_{2}+\ldots+n_{r}} \Gamma\left(\begin{array}{ccccc}
n_{r} & \ldots & n_{2} & n_{1} \\
z-a_{r} & \ldots & z-a_{2} & z-a_{1}
\end{array} ; z\right) .
$$


Formally reminiscent of the Hölder convolution in eq. (2.4), the above reflection identity is valid for all arguments $z \in \mathbb{C} \backslash\{0\}$. It can be proven using the parity properties of the weighting functions $f^{(n)}$ in eq. (2.17) and a reparametrization of the integration domain. If all the labels $a_{i}$ vanish, we will often use the notation

$$
\Gamma\left(n_{1}, n_{2}, \ldots, n_{r} ; z\right) \equiv \Gamma\left(\begin{array}{ccccc}
n_{1} & n_{2} & \ldots & n_{r} \\
0 & 0 & \ldots & 0
\end{array} ; z\right) .
$$

\subsubsection{Elliptic multiple zeta values}

Evaluating eIIs with all $a_{i}$ equal to 0 (or equivalently $a_{i}=1$ by the periodicity property eq. (2.14)) at $z=1$ gives rise to iterated integrals

$$
\begin{aligned}
\omega\left(n_{1}, n_{2}, \ldots, n_{r}\right) & \equiv \int_{0 \leq z_{i} \leq z_{i+1} \leq 1} f^{\left(n_{1}\right)}\left(z_{1}\right) \mathrm{d} z_{1} f^{\left(n_{2}\right)}\left(z_{2}\right) \mathrm{d} z_{2} \ldots f^{\left(n_{r}\right)}\left(z_{r}\right) \mathrm{d} z_{r} \\
& =\Gamma\left(n_{r}, \ldots, n_{2}, n_{1} ; 1\right)
\end{aligned}
$$

which we will refer to as elliptic multiple zeta values or eMZVs for short. They furnish a natural genus-one generalization of standard MZVs ${ }^{5}$ as defined in eq. (2.6). The shuffle relation eq. (2.19) can be straightforwardly applied to eMZVs

$$
\omega\left(n_{1}, n_{2}, \ldots, n_{r}\right) \omega\left(k_{1}, k_{2}, \ldots, k_{s}\right)=\omega\left(\left(n_{1}, n_{2}, \ldots, n_{r}\right) ш\left(k_{1}, k_{2}, \ldots, k_{s}\right)\right),
$$

and the parity property eq. $(2.17)$ of the functions $f^{(n)}$ implies the reflection identity

$$
\omega\left(n_{1}, n_{2}, \ldots, n_{r-1}, n_{r}\right)=(-1)^{n_{1}+n_{2}+\ldots+n_{r}} \omega\left(n_{r}, n_{r-1}, \ldots, n_{2}, n_{1}\right) .
$$

Note that a similar set of $\omega$ 's can be defined by an iterated integral along the path from 0 to $\tau$ replacing the integration domain $[0,1]$ in eq. (2.22). They appear in the modular transformations of eMZVs and naturally satisfy the properties eqs. (2.23) and (2.24) as well. Likewise, the eIIs defined in eq. (2.18) allow for a version with integrations on the path from 0 to $\tau$.

Regularization. Among the family of functions $f^{(n)}(z)$ used to define eIIs and eMZVs, only $f^{(1)}(z)$ has a simple pole at zero and its images under the translations in eq. (2.14). Therefore, iterated integrals of the form

$$
\Gamma\left(\begin{array}{ccccc}
n_{r} & \ldots & n_{2} & n_{1} \\
a_{r} & \ldots & a_{2} & a_{1}
\end{array} ; z\right)=\int_{0 \leq z_{i} \leq z_{i+1} \leq z} f^{\left(n_{1}\right)}\left(z_{1}-a_{1}\right) \mathrm{d} z_{1} f^{\left(n_{2}\right)}\left(z_{2}-a_{2}\right) \mathrm{d} z_{2} \ldots f^{\left(n_{r}\right)}\left(z_{r}-a_{r}\right) \mathrm{d} z_{r}
$$

with $n_{1}=1$ or $n_{r}=1$ need to be regularized if either $a_{1}=0$ or $a_{r}=z$. As with multiple polylogarithms, the idea is to slightly move the endpoints of the integration domain by a small parameter, and then to expand in this parameter. More precisely, one writes the integral

$$
\int_{\varepsilon \leq z_{i} \leq z_{i+1} \leq z-\varepsilon} f^{\left(n_{1}\right)}\left(z_{1}-a_{1}\right) \mathrm{d} z_{1} f^{\left(n_{2}\right)}\left(z_{2}-a_{2}\right) \mathrm{d} z_{2} \ldots f^{\left(n_{r}\right)}\left(z_{r}-a_{r}\right) \mathrm{d} z_{r}
$$

\footnotetext{
${ }^{5}$ In order to distinguish between eMZVs and MZVs, we will sometimes refer to the latter as standard MZVs.
} 
as a polynomial in $\ln (-2 \pi i \varepsilon)$, where the branch of the logarithm is chosen such that we have $\ln (-i)=-\frac{\pi i}{2}$. The regularized value of eq. (2.26) is then defined to be the constant term in this expansion. The additional $-2 \pi i$ in the expansion parameter $\ln (-2 \pi i \varepsilon)$ ensures that no logarithms appear in the limit $\tau \rightarrow i \infty$, and that eMZVs degenerate to MZVs. A thorough treatment of this degeneration can be found in ref. [24] and will be exploited in ref. [49].

\subsubsection{Removing the argument $z$ from the label}

As for the multiple polylogarithms, no arguments $z$ are allowed in the labels $\left\{a_{1} \ldots a_{r}\right\}$ in order to perform the integration using eq. (2.18). Therefore we need to find relations, which trade eIIs with one or multiple occurrences of the argument $z$ in the label for eIIs where $z$ appears in the argument exclusively. The key idea for finding those relations is to write the eII as the integral of its total derivative

$$
\Gamma\left(\begin{array}{cccccc}
n_{1} & n_{2} & \ldots & n_{q} & \ldots & n_{r} \\
a_{1} & a_{2} & \ldots & z & \ldots & a_{r}
\end{array} ; z\right)=\int_{0}^{z} \mathrm{~d} t \frac{\mathrm{d}}{\mathrm{d} t} \Gamma\left(\begin{array}{ccccccc}
n_{1} & n_{2} & \ldots & n_{q} & \ldots & n_{r} \\
a_{1} & a_{2} & \ldots & t & \ldots & \ldots & a_{r}
\end{array} ; t\right)+\lim _{z \rightarrow 0} \Gamma\left(\begin{array}{ccccccc}
n_{1} & n_{2} & \ldots & n_{q} & \ldots & n_{r} \\
a_{1} & a_{2} & \ldots & z & \ldots & a_{r}
\end{array} ; z\right)
$$

This resembles the strategy at genus zero which led to the identity eq. (2.11) between multiple polylogarithms. In the subsequent, we address additional features and subtleties intrinsic to the elliptic case. The feasibility of this approach in the elliptic scenario is discussed in ref. [16], see in particular theorem 26 therein.

Boundary terms. The boundary term at $z=0$ usually drops out from eq. (2.27) due to the vanishing volume of the integration domain. However, the special situation when all $n_{j}=1$ leads to the appearance of standard MZVs. As will be elaborated on in section 3, the function $f^{(1)}$ is the only source of singularities in the integration variables, as can be seen from its leading behavior $f^{(1)}(z)=z^{-1}+\mathcal{O}(z)$. Hence, the regime $z \rightarrow 0$ reproduces multiple polylogarithms as defined in eq. (2.1):

$$
\begin{aligned}
\lim _{z \rightarrow 0} \Gamma\left(\begin{array}{cccc}
1 & 1 & \ldots & 1 \\
a_{1} & a_{2} & \ldots & a_{r}
\end{array} ; z\right) & =\lim _{z \rightarrow 0} \int_{0}^{z} \frac{\mathrm{d} t_{1}}{t_{1}-a_{1}} \int_{0}^{t_{1}} \frac{\mathrm{d} t_{2}}{t_{2}-a_{2}} \ldots \int_{0}^{t_{r-1}} \frac{\mathrm{d} t_{r}}{t_{r}-a_{r}} \\
& =\lim _{z \rightarrow 0} G\left(a_{1}, a_{2}, \ldots, a_{r} ; z\right) .
\end{aligned}
$$

If all $a_{j} \in\{0, z\}$, the scaling relation eq. (2.3) allows to rewrite the polylogarithms in terms of MZVs (see eq. (2.6)), leading to

$$
\lim _{z \rightarrow 0} \Gamma\left(\begin{array}{cccc}
n_{1} & n_{2} & \ldots & n_{r} \\
b_{1} z & b_{2} z & \ldots & b_{r} z
\end{array} ; z\right)=G\left(b_{1}, b_{2}, \ldots, b_{r} ; 1\right) \prod_{j=1}^{r} \delta_{n_{j}, 1}, \quad b_{j} \in\{0,1\} .
$$

Partial derivatives. The total $t$-derivative in eq. (2.27) can be written in terms of partial derivatives with respect to the arguments and the labels. This requires the elliptic analogues of eqs. (A.1) to (A.3) listed below in order to arrive at shorter elliptic polylogarithms. The derivative with respect to the argument

$$
\frac{\partial}{\partial z} \Gamma\left(\begin{array}{cccc}
n_{1} & n_{2} & \ldots & n_{r} \\
a_{1} & a_{2} & \ldots & a_{r}
\end{array} ; z\right)=f^{\left(n_{1}\right)}\left(z-a_{1}\right) \Gamma\left(\begin{array}{cccc}
n_{2} & \ldots & n_{r} \\
a_{2} & \ldots & a_{r}
\end{array} ; z\right)
$$


follows straightforwardly from eq. (2.18). Slightly more work using $\frac{\partial}{\partial a} f^{(n)}(t-a)=$ $-\frac{\partial}{\partial t} f^{(n)}(t-a)$ as well as eq. (2.30) is required for derivatives with respect to labels $a_{q}$. Starting with the special cases $q=1$ and $q=r$ one finds

$$
\begin{aligned}
& \frac{\partial}{\partial a_{1}} \Gamma\left(\begin{array}{cccc}
n_{1} & n_{2} & \ldots & n_{r} \\
a_{1} & a_{2} & \ldots & a_{r}
\end{array} ; t_{0}\right)=-f^{\left(n_{1}\right)}\left(t_{0}-a_{1}\right) \Gamma\left(\begin{array}{ccccc}
n_{2} & n_{3} & \ldots & n_{r} \\
a_{2} & a_{3} & \ldots & a_{r}
\end{array} ; t_{0}\right) \\
& +\int_{0}^{t_{0}} \mathrm{~d} t f^{\left(n_{1}\right)}\left(t-a_{1}\right) f^{\left(n_{2}\right)}\left(t-a_{2}\right) \Gamma\left(\begin{array}{cccc}
n_{3} & \ldots & n_{r} \\
a_{3} & \ldots & a_{r}
\end{array} ; t\right) \\
& \frac{\partial}{\partial a_{r}} \Gamma\left(\begin{array}{cccc}
n_{1} & n_{2} & \ldots & n_{r} \\
a_{1} & a_{2} & \ldots & a_{r}
\end{array} ; t_{0}\right)=f^{\left(n_{r}\right)}\left(-a_{r}\right) \Gamma\left(\begin{array}{ccccc}
n_{1} & n_{2} & \ldots & n_{r-1} \\
a_{1} & a_{2} & \ldots & a_{r-1}
\end{array} ; t_{0}\right) \\
& -\left(\prod_{j=1}^{r-2} \int_{0}^{t_{j-1}} \mathrm{~d} t_{j} f^{\left(n_{j}\right)}\left(t_{j}-a_{j}\right)\right) \int_{0}^{t_{r-2}} \mathrm{~d} t f^{\left(n_{r-1}\right)}\left(t-a_{r-1}\right) f^{\left(n_{r}\right)}\left(t-a_{r}\right) .
\end{aligned}
$$

Deriving with respect to a label $a_{q}$ with $q \neq 1, r$ yields

$$
\begin{aligned}
& \frac{\partial}{\partial a_{q}} \Gamma\left(\begin{array}{ccccc}
n_{1} & n_{2} & \ldots & n_{r} \\
a_{1} & a_{2} & \ldots & a_{r}
\end{array} ; t_{0}\right) \\
& =\left(\prod_{j=1}^{q-1} \int_{0}^{t_{j-1}} \mathrm{~d} t_{j} f^{\left(n_{j}\right)}\left(t_{j}-a_{j}\right)\right) \int_{0}^{t_{q-1}} \mathrm{~d} t f^{\left(n_{q}\right)}\left(t-a_{q}\right) f^{\left(n_{q+1}\right)}\left(t-a_{q+1}\right) \Gamma\left(\begin{array}{ccc}
n_{q+2} & \ldots & n_{r} \\
a_{q+2} & \ldots & a_{r}
\end{array} ;\right) \\
& -\left(\prod_{j=1}^{q-2} \int_{0}^{t_{j-1}} \mathrm{~d} t_{j} f^{\left(n_{j}\right)}\left(t_{j}-a_{j}\right)\right) \int_{0}^{t_{q-2}} \mathrm{~d} t f^{\left(n_{q-1}\right)}\left(t-a_{q-1}\right) f^{\left(n_{q}\right)}\left(t-a_{q}\right) \Gamma\left(\begin{array}{ccc}
n_{q+1} & \ldots & n_{r} \\
a_{q+1} & \ldots & a_{r}
\end{array} ;\right) .
\end{aligned}
$$

Total derivatives. Summing the above partial derivatives with respect to the argument $z$ and the labels $a_{q}$, total derivatives from eq. (2.27) can be expressed in a very efficient way. For a single instance of $a_{q}=z$, the special cases $q=1$ and $q=r$ give rise to

$$
\begin{gathered}
\frac{\mathrm{d}}{\mathrm{d} t_{0}} \Gamma\left(\begin{array}{cccc}
n_{1} & n_{2} & \ldots & n_{r} \\
t_{0} & a_{2} & \ldots & a_{r}
\end{array} ; t_{0}\right)=\int_{0}^{t_{0}} \mathrm{~d} t f^{\left(n_{1}\right)}\left(t-t_{0}\right) f^{\left(n_{2}\right)}\left(t-a_{2}\right) \Gamma\left(\begin{array}{cccc}
n_{3} & \ldots & n_{r} \\
a_{3} & \ldots & a_{r}
\end{array} ;\right) \quad \text { and } \\
\frac{\mathrm{d}}{\mathrm{d} t_{0}} \Gamma\left(\begin{array}{llll}
n_{1} & \ldots & n_{r-1} & n_{r} \\
a_{1} & \ldots & a_{r-1} & t_{0}
\end{array} ; t_{0}\right)=f^{\left(n_{1}\right)}\left(t_{0}-a_{1}\right) \Gamma\left(\begin{array}{cccc}
n_{2} & \ldots & n_{r-1} & n_{r} \\
a_{2} & \ldots & a_{r-1} & t_{0}
\end{array} ; t_{0}\right)+f^{\left(n_{r}\right)}\left(-t_{0}\right) \Gamma\left(\begin{array}{ccc}
n_{1} & \ldots & n_{r-1} \\
a_{1} & \ldots & a_{r-1}
\end{array} t_{0}\right) \\
\quad-\left(\prod_{j=1}^{t_{0}} \int_{0}^{t_{j-1}} \mathrm{~d} t_{j} f^{\left(n_{j}\right)}\left(t_{j}-a_{j}\right)\right) \int_{0}^{t_{r-2}} \mathrm{~d} t f^{\left(n_{r-1}\right)}\left(t-a_{r-1}\right) f^{\left(n_{r}\right)}\left(t-t_{0}\right) .
\end{gathered}
$$

For $q \neq 1, r$, the integrand of eq. (2.27) takes the form

$$
\begin{aligned}
& \frac{\mathrm{d}}{\mathrm{d} t_{0}} \Gamma\left(\begin{array}{cccccccc}
n_{1} & n_{2} & \ldots & n_{q-1} & n_{q} & n_{q+1} & \ldots & n_{r} \\
a_{1} & a_{2} & \ldots & a_{q-1} & t_{0} & a_{q+1} & \ldots & a_{r}
\end{array} ; t_{0}\right)=f^{\left(n_{1}\right)}\left(t_{0}-a_{1}\right) \Gamma\left(\begin{array}{ccccccc}
n_{2} & \ldots & n_{q-1} & n_{q} & n_{q+1} & \ldots & n_{r} \\
a_{2} & \ldots & a_{q-1} & t_{0} & a_{q+1} & \ldots & a_{r}
\end{array} ; t_{0}\right) \\
& +\left(\prod_{j=1}^{q-1} \int_{0}^{t_{j-1}} \mathrm{~d} t_{j} f^{\left(n_{j}\right)}\left(t_{j}-a_{j}\right)\right) \int_{0}^{t_{q-1}} \mathrm{~d} t f^{\left(n_{q}\right)}\left(t-t_{0}\right) f^{\left(n_{q+1}\right)}\left(t-a_{q+1}\right) \Gamma\left(\begin{array}{ccc}
n_{q+2} & \ldots & n_{r} \\
a_{q+2} & \ldots & a_{r}
\end{array} ;\right) \\
& -\left(\prod_{j=1}^{q-2} \int_{0}^{t_{j-1}} \mathrm{~d} t_{j} f^{\left(n_{j}\right)}\left(t_{j}-a_{j}\right)\right) \int_{0}^{t_{q-2}} \mathrm{~d} t f^{\left(n_{q-1}\right)}\left(t-a_{q-1}\right) f^{\left(n_{q}\right)}\left(t-t_{0}\right) \Gamma\left(\begin{array}{lll}
n_{q+1} & \ldots & n_{r} \\
a_{q+1} \ldots & a_{r}
\end{array} ;\right) .
\end{aligned}
$$

Further examples with repeated appearances of $t_{0}$ are displayed in appendix B.1. 
Fay identities. Having applied the above derivative identities, one is usually left with expressions containing integrals of the form

$$
\int_{0}^{z} \mathrm{~d} t f^{\left(n_{1}\right)}\left(t-a_{1}\right) f^{\left(n_{2}\right)}\left(t-a_{2}\right)
$$

where the integration variable appears in the argument of more than one function $f^{(n)}$. In the corresponding situation for multiple polylogarithms, with weights of the form eq. (2.13), one would have used partial fraction identities

$$
\frac{1}{(t-a)(t-b)}=\frac{1}{(t-a)(a-b)}+\frac{1}{(t-b)(b-a)}
$$

in order to avoid the repeated appearance of the integration variable $t$. Analogous relations for the more general class of weighting functions $f^{(n)}$ are provided by Fay identities, which will be put in a larger mathematical context in section 3 below. They relate products $f^{\left(n_{1}\right)} f^{\left(n_{2}\right)}$ at arguments $x, t$ and $x-t$ and thereby allow to systematically remove repeated appearances of some integration variable. A simple example of a Fay identity relates products of functions $f^{(1)}$ to a sum of functions $f^{(2)}$

$$
f^{(1)}(t-x) f^{(1)}(t)=f^{(1)}(t-x) f^{(1)}(x)-f^{(1)}(t) f^{(1)}(x)+f^{(2)}(t)+f^{(2)}(x)+f^{(2)}(t-x) .
$$

The general relation, which is valid for complex arguments $x, t$ as well,

$$
\begin{aligned}
f^{\left(n_{1}\right)}(t-x) f^{\left(n_{2}\right)}(t)=- & (-1)^{n_{1}} f^{\left(n_{1}+n_{2}\right)}(x)+\sum_{j=0}^{n_{2}}\left(\begin{array}{c}
n_{1}-1+j \\
j
\end{array}\right) f^{\left(n_{2}-j\right)}(x) f^{\left(n_{1}+j\right)}(t-x) \\
& +\sum_{j=0}^{n_{1}}\left(\begin{array}{c}
n_{2}-1+j \\
j
\end{array}\right)(-1)^{n_{1}+j} f^{\left(n_{1}-j\right)}(x) f^{\left(n_{2}+j\right)}(t)
\end{aligned}
$$

in turn allows to remove all repeated occurrences of the variable $t$. Iterating the above steps, one can thus eliminate all arguments from the label of any eII recursively.

Result. Combining the Fay identity eq. (2.40) with the total derivatives in eqs. (2.34) to (2.36) turns (2.27) into a recursive rule for removing the argument $z$ from the label of $\Gamma\left(\begin{array}{ccccc}n_{1} & \ldots & n_{q} & \ldots & n_{r} \\ a_{1} & \ldots & z & \ldots & a_{r}\end{array} ; z\right)$. In the equations below, all terms on the right-hand side are either free of $a_{q}=z$ or have shorter labels. The special cases $q=1$ and $q=r$ yield

$$
\begin{aligned}
& \Gamma\left(\begin{array}{cccc}
n_{1} & n_{2} & \ldots & n_{r} \\
z & a_{2} & \ldots & a_{r}
\end{array} ; z\right)=\lim _{z \rightarrow 0} G\left(z, a_{2}, \ldots, a_{r} ; z\right) \prod_{j=1}^{r} \delta_{n_{j}, 1}-(-1)^{n_{1}} \Gamma\left(\begin{array}{cccccc}
n_{1}+n_{2} & 0 & n_{3} & \ldots & n_{r} \\
a_{2} & 0 & 0 & a_{3} & \ldots & a_{r}
\end{array} \quad z\right) \\
& +\sum_{j=0}^{n_{1}}(-1)^{n_{1}+j}\left(\begin{array}{c}
n_{2}-1+j \\
j
\end{array}\right) \Gamma\left(\begin{array}{ccccc}
n_{1}-j & n_{2}+j & n_{3} & \ldots & n_{r} \\
a_{2} & a_{2} & a_{3} & \ldots & a_{r}
\end{array} ;\right) \\
& +\sum_{j=0}^{n_{2}}\left(\begin{array}{c}
n_{1}-1+j \\
j
\end{array}\right) \int_{0}^{z} \mathrm{~d} t f^{\left(n_{2}-j\right)}\left(t-a_{2}\right) \Gamma\left(\begin{array}{ccccc}
n_{1}+j & n_{3} & \ldots & n_{r} \\
t & a_{3} & \ldots & a_{r}
\end{array} ;\right) \\
& \Gamma\left(\begin{array}{ccccc}
n_{1} & \ldots & n_{r-1} & n_{r} \\
a_{1} & \ldots & a_{r-1} & z
\end{array} ; z\right)=\lim _{z \rightarrow 0} G\left(a_{1}, \ldots, a_{r-1}, z ; z\right) \prod_{j=1}^{r} \delta_{n_{j}, 1}+\int_{0}^{z} \mathrm{~d} t f^{\left(n_{1}\right)}\left(t-a_{1}\right) \Gamma\left(\begin{array}{ccccc}
n_{2} & \ldots & n_{r-1} & n_{r} \\
a_{2} & \ldots & a_{r-1} & t
\end{array} ; t\right) \\
& +(-1)^{n_{r}} \Gamma\left(\begin{array}{cccc}
n_{r} & n_{1} & \ldots & n_{r-1} \\
0 & a_{1} & \ldots & a_{r-1}
\end{array} ; z\right)+(-1)^{n_{r}} \Gamma\left(\begin{array}{cccccc}
n_{r-1}+n_{r} & n_{1} & \ldots & n_{r-2} & 0 \\
a_{r-1} & a_{1} & \ldots & a_{r-2} & 0
\end{array} ; z\right)
\end{aligned}
$$




$$
\begin{aligned}
& -\sum_{j=0}^{n_{r-1}}\left(\begin{array}{c}
n_{r}-1+j \\
j
\end{array}\right) \int_{0}^{z} \mathrm{~d} t f^{\left(n_{r-1}-j\right)}\left(t-a_{r-1}\right) \Gamma\left(\begin{array}{cccc}
n_{1} & \ldots & n_{r-2} \\
a_{1} & \ldots & a_{r-2} & n_{r}+j
\end{array} ; t\right) \\
& -\sum_{j=0}^{n_{r}}\left(\begin{array}{c}
n_{r-1}-1+j \\
j
\end{array}\right)(-1)^{n_{r}+j} \Gamma\left(\begin{array}{cccccc}
n_{r}-j & n_{1} & \ldots & n_{r-2} & n_{r-1}+j \\
a_{r-1} & a_{1} & \ldots & a_{r-2} & a_{r-1}
\end{array} ;\right),
\end{aligned}
$$

while $a_{q}=z$ at a generic position $q \neq 1, r$ can be addressed via

$$
\begin{aligned}
& \Gamma\left(\begin{array}{cccccccc}
n_{1} & n_{2} & \ldots & n_{q-1} & n_{q} & n_{q+1} & \ldots & n_{r} \\
a_{1} & a_{2} & \ldots & a_{q-1} & a_{q} & a_{q+1} & \ldots & a_{r}
\end{array} ; z\right)=\lim _{z \rightarrow 0} G\left(a_{1}, \ldots, a_{q-1}, z, a_{q+1}, \ldots, a_{r} ; z\right) \prod_{j=1}^{r} \delta_{n_{j}, 1} \\
& +\int_{0}^{z} \mathrm{~d} t f^{\left(n_{1}\right)}\left(t-a_{1}\right) \Gamma\left(\begin{array}{ccccccc}
n_{2} & \ldots & n_{q-1} & n_{q} & n_{q+1} & \ldots & n_{r} \\
a_{2} & \ldots & a_{q-1} & t & a_{q+1} & \ldots & a_{r}
\end{array} ; t\right) \\
& -(-1)^{n_{q}} \Gamma\left(\begin{array}{cccccccccc}
n_{q}+n_{q+1} & n_{1} & \ldots & n_{q-1} & 0 & n_{q+2} & \ldots & n_{r} \\
a_{q+1} & a_{1} & \ldots & a_{q-1} & 0 & a_{q+2} & \ldots & a_{r}
\end{array} ; z\right)+(-1)^{n_{q}} \Gamma\left(\begin{array}{ccccccccc}
n_{q}+n_{q-1} & n_{1} & \ldots & n_{q-2} & 0 & n_{q+1} & \ldots & n_{r} \\
a_{q-1} & a_{1} & \ldots & a_{q-2} & 0 & a_{q+1} & \ldots & a_{r}
\end{array} ; z\right)
\end{aligned}
$$

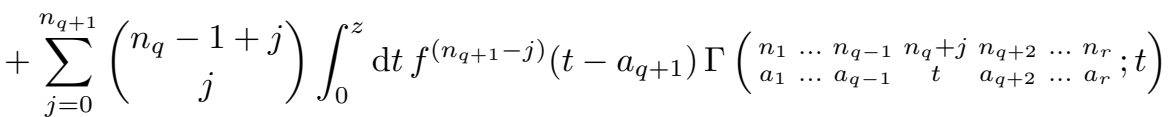

$$
\begin{aligned}
& +\sum_{j=0}^{n_{q}}\left(\begin{array}{c}
n_{q+1}-1+j \\
j
\end{array}\right)(-1)^{n_{q}+j} \Gamma\left(\begin{array}{ccccccccc}
n_{q}-j & n_{1} & \ldots & n_{q-1} & n_{q+1}+j & n_{q+2} & \ldots & \\
a_{q+1} & a_{1} & \ldots & a_{q-1} & a_{q+1} & a_{q+2} & \ldots & a_{r}
\end{array} ; z\right) \\
& -\sum_{j=0}^{n_{q-1}}\left(\begin{array}{c}
n_{q}-1+j \\
j
\end{array}\right) \int_{0}^{z} \mathrm{~d} t f^{\left(n_{q-1}-j\right)}\left(t-a_{q-1}\right) \Gamma\left(\begin{array}{ccccccc}
n_{1} & \ldots & n_{q-2} \\
a_{1} & \ldots & a_{q-2} & n_{q}+j & n_{q+1} & \ldots & n_{r} \\
a_{q+1} & \ldots & a_{r}
\end{array} ; t\right)
\end{aligned}
$$

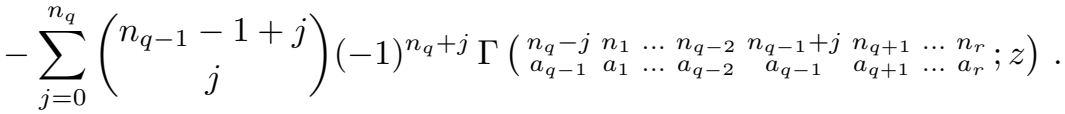

Situations with multiple successive appearance of $a_{j}=z$ are discussed in appendix B.

Examples. At length one, the reflection identity eq. (2.20) implies that

$$
\Gamma\left({ }_{z}^{n} ; z\right)=(-1)^{n} \Gamma(n ; z),
$$

which covers all identities at this length. At length two, cases with $n_{1}=0$ or $n_{2}=0$ are similarly determined by eq. (2.20), so the simplest non-trivial application of eq. (2.27)

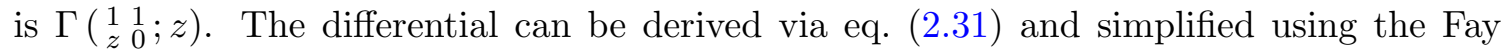
identity eq. (2.39) as well as eq. (2.44),

$$
\frac{\mathrm{d}}{\mathrm{d} t} \Gamma\left(\begin{array}{cc}
1 & 1 \\
t & 0
\end{array} t\right)=2 \Gamma(2 ; t)+f^{(2)}(t) \Gamma(0 ; t)-2 f^{(1)}(t) \Gamma(1 ; t),
$$

see eq. (2.21) for the notation on the right hand side. In combination with the boundary term

$$
\lim _{z \rightarrow 0} \Gamma\left(\underset{z}{1} \frac{1}{0} ; z\right)=G(1,0 ; 1)=\zeta_{2}
$$

we find

$$
\Gamma\left({ }_{z}^{1} \frac{1}{0} ; z\right)=2 \Gamma(0,2 ; z)+\Gamma(2,0 ; z)-2 \Gamma(1,1 ; z)+\zeta_{2},
$$

which of course agrees with the general formula eq. (2.41). The same reasoning can be applied recursively to obtain for example

$$
\Gamma\left(\begin{array}{ccc}
1 & 1 & 1 \\
z & 0 & 0
\end{array} ; z\right)=-\Gamma\left(\begin{array}{ccc}
1 & 1 & 1 \\
z & z & 0
\end{array} ; z\right)=-\Gamma(0,3,0 ; z)-\Gamma(0,0,3 ; z)-3 \Gamma(1,1,1 ; z)+\Gamma(2,0,1 ; z)
$$




$$
\begin{array}{r}
+\Gamma(1,2,0 ; z)+2 \Gamma(0,2,1 ; z)+2 \Gamma(1,0,2 ; z)+\zeta_{2} \Gamma(1 ; z)-\zeta_{3} \\
\Gamma\left(\begin{array}{cccc}
1 & 0 & 0 & 1 \\
z & 0 & 0 & 0
\end{array} ; z\right)=2 \Gamma(0,0,0,2 ; z)+\Gamma(0,0,2,0 ; z)-2 \Gamma(0,0,1,1 ; z)+\zeta_{2} \Gamma(0,0 ; z)
\end{array}
$$

as well as

$$
\begin{aligned}
& \Gamma\left(\begin{array}{ccccc}
0 & 1 & 0 & 1 & 0 \\
0 & z & 0 & 0 & 0
\end{array} ; z\right)=2 \Gamma(0,0,0,2,0 ; z)+\Gamma(0,2,0,0,0 ; z)-2 \Gamma(0,1,0,1,0 ; z) \\
& \Gamma\left(\begin{array}{ccccc}
0 & 1 & 1 & 0 & 0
\end{array} 0 ; z\right)=\Gamma(0,0,2,0,0 ; z)+\Gamma(0,0,0,2,0 ; z)+\Gamma(2,0,0,0,0 ; z) \\
& -\Gamma(1,0,1,0,0 ; z)-\Gamma(1,0,0,1,0 ; z) .
\end{aligned}
$$

In subsection 4.3 these relations turn out to be crucial to express the low energy expansion of one-loop string amplitudes in terms of eMZVs.

The most general relation at length two following from eq. (2.41) reads

$$
\begin{aligned}
& \Gamma\left(\begin{array}{cc}
n_{1} & n_{2} \\
z & 0
\end{array} z\right)=-(-1)^{n_{1}} \Gamma\left(n_{1}+n_{2}, 0 ; z\right)+\sum_{r=0}^{n_{2}}(-1)^{n_{1}+r}\left(\begin{array}{c}
n_{1}-1+r \\
r
\end{array}\right) \Gamma\left(n_{2}-r, n_{1}+r ; z\right) \\
& +\sum_{r=0}^{n_{1}}(-1)^{n_{1}+r}\left(\begin{array}{c}
n_{2}-1+r \\
r
\end{array}\right) \Gamma\left(n_{1}-r, n_{2}+r ; z\right)+\delta_{n_{1}, 1} \delta_{n_{2}, 1} \zeta_{2},
\end{aligned}
$$

and determines $\Gamma\left(\begin{array}{cc}n_{1} & n_{2} \\ 0 & z\end{array} ;\right)$ through the shuffle identity and eq. (2.44). Analogous relations at length three can be found in appendix B.3.

\subsubsection{Relations among elliptic multiple zeta values}

Apart from their application to string amplitudes, the above manipulations of eIIs are instrumental to derive relations among eMZVs beyond the obvious reflection and shuffle properties. By definition eq. (2.22), eIIs with all labels $a_{j}=0$ yield eMZVs in the limit $z \rightarrow 1$ of their argument. At the level of labels $a_{j}=z$, the limit $z \rightarrow 1$ is equivalent to $a_{j} \rightarrow 0$ since the $f^{(n)}$ are periodic under $z \mapsto z+1$, hence

$$
\lim _{z \rightarrow 1} \Gamma\left(\begin{array}{cccc}
n_{1} & n_{2} & \ldots & n_{r} \\
a_{1} & a_{2} & \ldots & a_{r}
\end{array} ; z\right)=\omega\left(n_{r}, \ldots, n_{2}, n_{1}\right), \quad a_{j} \in\{0, z\}, \quad n_{1}, n_{r} \neq 1
$$

Note that endpoint divergences caused by the simple pole in $f^{(1)}$ might introduce additional MZV constants similar to eq. (2.29), that is why the cases $n_{1}, n_{r}=1$ are excluded explicitly.

At length two, for example, eq. (2.52) implies the following eMZV identity provided that the limit $z \rightarrow 1$ is non-singular:

$$
\begin{aligned}
\omega\left(n_{2}, n_{1}\right)= & -(-1)^{n_{1}} \omega\left(0, n_{1}+n_{2}\right)+\sum_{r=0}^{n_{2}}(-1)^{n_{1}+r}\left(\begin{array}{c}
n_{1}-1+r \\
r
\end{array}\right) \omega\left(n_{1}+r, n_{2}-r\right) \\
& +\sum_{r=0}^{n_{1}}(-1)^{n_{1}+r}\left(\begin{array}{c}
n_{2}-1+r \\
r
\end{array}\right) \omega\left(n_{2}+r, n_{1}-r\right), \quad n_{1}, n_{2} \neq 1 .
\end{aligned}
$$

At low weights $n_{i}$, the coefficients in eq. (2.54) are particularly simple such as

$$
\omega(2,3)=\omega(0,5), \quad \omega(3,4)=-2 \omega(0,7)+\omega(2,5) .
$$


Similar procedures can be carried out at higher length. Combining e.g. eq. (B.7) and a suitable generalization thereof to length four leads to

$$
\begin{aligned}
& 0=\omega(0,0,5)+\omega(0,1,4)+\omega(2,0,3) \\
& 0=10 \omega(0,0,0,5)+4 \omega(0,0,3,2)+2 \omega(0,2,0,3)-\omega(2) \omega(0,3)-\omega(0,5)
\end{aligned}
$$

At length five, a combination of eqs. (2.50) and (2.51) with the shuffle relation eq. (2.23) yields

$$
\begin{aligned}
& \omega(0,1,0,1,0)=\omega(0,2,0,0,0) \\
& \omega(0,1,1,0,0)=\omega(2,0,0,0,0)-\omega(2) \omega(0,0,0,0),
\end{aligned}
$$

which will be applied in subsection 4.3 .

\section{The functions $f^{(n)}$ on the elliptic curve}

In this section, we provide the definition and mathematical framework for the functions $f^{(n)}$, thereby supplementing our heuristic approach in section 2. Before doing so, let us start with some mathematical motivation, in which we explain in particular why we need — in distinction to multiple polylogarithms — an infinite number of them.

\subsection{Motivation}

The importance of multiple polylogarithms as defined in eq. (2.1) becomes evident, when considering homotopy-invariant iterated integrals on the multiply punctured complex plane $\mathbb{C} \backslash\left\{a_{1}, \ldots, a_{n}\right\}$ : the value of any such integral evaluated on a path $\gamma$ depends on the homotopy class of the path only and is a $\mathbb{C}$-linear combination of multiple polylogarithms.

Instead of the multiply punctured plane, let us now consider the complex elliptic curve $E_{\tau}=\mathbb{C} /(\mathbb{Z}+\mathbb{Z} \tau)$ with its origin removed (we write this as $E_{\tau}^{\times}$), where $\operatorname{Im}(\tau)>0$. One possible definition of multiple elliptic polylogarithms is via iterated integrals on $E_{\tau}^{\times}$. Writing the canonical coordinate on $E_{\tau}^{\times}$as $z=s+r \tau$ with $s, r \in \mathbb{R}$, such that $r \equiv \frac{\operatorname{Im}(z)}{\operatorname{Im}(\tau)}$, two natural differential forms on $E_{\tau}^{\times}$read

$$
\mathrm{d} z \quad \text { and } \quad \nu \equiv 2 \pi i \mathrm{~d} r .
$$

These differential forms, however, are not sufficient to describe all iterated integrals on $E_{\tau}^{\times}$. Even worse, iterated integrals employing the differential forms $\mathrm{d} z$ and $\nu$ only will not be homotopy-invariant in general, i.e. they will depend on the choice of a path in a given homotopy class.

Both problems are overcome simultaneously by supplementing eq. (3.1) by an infinite tower of differentials $f^{(n)}(z) \mathrm{d} z$ constructed through a generating function $[16]^{6}$

$$
\Omega(z, \alpha, \tau)=\sum_{n \geq 0} f^{(n)}(z) \alpha^{n-1}
$$

\footnotetext{
${ }^{6}$ Note that in ref. [16], $\Omega(z, \alpha, \tau)$ is defined as a differential form, i.e. includes $\mathrm{d} z$.
} 
where $f^{(0)}(z) \equiv 1$. In particular, it has been proven in ref. [16] that every iterated integral in $\nu$ and $\mathrm{d} z$ can be uniquely lifted to a homotopy-invariant iterated integral over $\nu$ and $f^{(n)}(z) \mathrm{d} z$. Conversely, every homotopy-invariant iterated integral on $E_{\tau}^{\times}$arises in this way.

The form of the generating function and its coefficients $f^{(n)}$ in eq. (3.2) can be fixed by constructing a doubly-periodic connection $J$ satisfying the integrability condition

$$
\mathrm{d} J+J \wedge J=0 .
$$

This requirement singles out a unique completion of $J=\nu X_{0}+\mathrm{d} z X_{1}+\ldots$ to a formal power series in non-commuting variables $X_{0}$ and $X_{1}$ given by [16]

$$
J=\nu X_{0}-\operatorname{ad}_{X_{0}} \Omega\left(z,-\operatorname{ad}_{X_{0}}, \tau\right)\left(X_{1}\right) \mathrm{d} z .
$$

It follows from eq. (3.3) that every word in $X_{0}, X_{1}$ in the formal power series

$$
\sum_{k=0}^{\infty} \int J^{k}
$$

is a homotopy-invariant iterated integral on $E_{\tau}^{\times}$, and one can prove that in fact every such iterated integral arises in this way. Therefore, every homotopy invariant iterated integral on $E_{\tau}^{\times}$can be written as a special linear combination of iterated integrals of the differential forms $f^{(n)}(z) \mathrm{d} z$ and $\nu$. The differential form $\nu$ eq. (3.1), however, vanishes on the real integration path $\gamma(t) \in \mathbb{R}$. Hence, the setup in subsection 2.2 based on real variables leads to elliptic multiple zeta values defined in ref. [17] without referring to the differential form $\nu$.

Although homotopy invariance is generically lost for the iterated integral over the forms $f^{\left(n_{1}\right)}\left(z_{1}\right) \mathrm{d} z_{1} \ldots f^{\left(n_{r}\right)}\left(z_{r}\right) \mathrm{d} z_{r}$ on the punctured elliptic curve $E_{\tau}^{\times}$, its value at the real path $[0,1]$ as in eq. $(2.22)$ can in fact be written as a $\mathbb{Z}$-linear combination of coefficients of words in eq. (3.5), again evaluated on the path $[0,1]$. In particular, this shows that the eMZVs associated with the path $[0,1][17]$ are periods of the fundamental group of $E_{\tau}^{\times}$.

Hence, the eIIs defined by eq. (2.18) coincide with the elliptic polylogarithms defined in ref. [16] when restricted to the real line. They can be lifted to honest homotopy-invariant iterated integrals on the punctured elliptic curve by means of the differential form $\nu$ defined in eq. (3.1). However, generic combinations of $f^{(n)}(z) \mathrm{d} z$ accompany several words in $X_{0}, X_{1}$ in eq. (3.5) and therefore allow for various homotopy-invariant completions using $\nu$. Iterated integrals over $\nu$ and $\mathrm{d} z$, on the other hand, correspond to a single word in eq. (3.5) and therefore have a unique uplift via $f^{(n \geq 1)}(z) \mathrm{d} z$ towards the elliptic polylogarithms of ref. [16].

\subsection{Doubly-periodic functions and generating series}

In this section, we define the functions $f^{(n)}$ through a generating series, closely following ref. [16]. In the sequel, $z$ and $\alpha$ are complex coordinates on $E_{\tau}^{\times}$. Simultaneously, $\alpha$ will be used as a formal expansion variable below. The modular parameter often appears in the combination

$$
q \equiv e^{2 \pi i \tau},
$$

where $\operatorname{Im}(\tau)>0$ translates into $|q|<1$, relevant for convergence issues. 


\subsubsection{Some doubly-periodic functions}

A general reference on doubly-periodic functions is ref. [23]. Let $\theta_{1}$ denote the odd Jacobi function ${ }^{7}$ defined by

$$
\theta_{1}(z, \tau) \equiv 2 i q^{1 / 8} \sin (\pi z) \prod_{j=1}^{\infty}\left(1-q^{j}\right) \prod_{j=1}^{\infty}\left(1-e^{2 \pi i z} q^{j}\right) \prod_{j=1}^{\infty}\left(1-e^{-2 \pi i z} q^{j}\right),
$$

subject to the following periodicity properties

$$
\theta_{1}(z+1, \tau)=-\theta_{1}(z, \tau), \quad \theta_{1}(z+\tau, \tau)=-e^{-\pi i \tau} e^{-2 \pi i z} \theta_{1}(z, \tau) .
$$

For $j \geq 1$ we also define the Eisenstein function $E_{j}(z, \tau)$ and the Eisenstein series $e_{j}(\tau)$ by $^{8}$

$$
E_{j}(z, \tau) \equiv \sum_{m, n \in \mathbb{Z}} \frac{1}{(z+m+n \tau)^{j}} \quad e_{j}(\tau) \equiv \sum_{\substack{m, n \in \mathbb{Z} \\(m, n) \neq(0,0)}} \frac{1}{(m+n \tau)^{j}}
$$

which are related to the function $\theta_{1}(z, \tau)$ via

$$
\frac{\partial}{\partial z} \ln \left(\theta_{1}(z, \tau)\right)=E_{1}(z, \tau), \quad \frac{\partial}{\partial z} E_{j}(z, \tau)=-j E_{j+1}(z, \tau) .
$$

\subsubsection{The Eisenstein-Kronecker series}

The Eisenstein-Kronecker series $F(z, \alpha, \tau)$ is defined by $[16,51]$

$$
F(z, \alpha, \tau) \equiv \frac{\theta_{1}^{\prime}(0, \tau) \theta_{1}(z+\alpha, \tau)}{\theta_{1}(z, \tau) \theta_{1}(\alpha, \tau)},
$$

where ' denotes a derivative with respect to the first argument. Taking the logarithmic derivative of eq. (3.11) together with the Taylor expansion $E_{1}(\alpha, \tau)=\frac{1}{\alpha}-\sum_{j=0}^{\infty} \alpha^{j} e_{j+1}(\tau)$ leads to the following alternative representation $[50,52]$

$$
F(z, \alpha, \tau)=\frac{1}{\alpha} \exp \left(-\sum_{j \geq 1} \frac{(-\alpha)^{j}}{j}\left(E_{j}(z, \tau)-e_{j}(\tau)\right)\right)
$$

in terms of the Eisenstein functions and Eisenstein series defined in eq. (3.9). The periodicity properties of the $\theta_{1}$-function in eq. (3.8) imply that the Eisenstein-Kronecker series is quasi-periodic,

$$
F(z+1, \alpha, \tau)=F(z, \alpha, \tau), \quad F(z+\tau, \alpha, \tau)=e^{-2 \pi i \alpha} F(z, \alpha, \tau) .
$$

Moreover, the representation (3.12) together with the Fay trisecant equation [53] yields the Fay identity

$$
\begin{aligned}
F\left(z_{1}, \alpha_{1}, \tau\right) F\left(z_{2}, \alpha_{2}, \tau\right)= & F\left(z_{1}, \alpha_{1}+\alpha_{2}, \tau\right) F\left(z_{2}-z_{1}, \alpha_{2}, \tau\right) \\
& +F\left(z_{2}, \alpha_{1}+\alpha_{2}, \tau\right) F\left(z_{1}-z_{2}, \alpha_{1}, \tau\right) .
\end{aligned}
$$

\footnotetext{
${ }^{7}$ The subsequent definitions of $f^{(n)}$ are unchanged by $z$-independent rescalings of $\theta_{1}$. Hence, the current setup is consistent with refs. [16, 50], which rely on $\theta(z, \tau) \equiv 2 i q^{1 / 12} \sin (\pi z) \prod_{j=1}^{\infty}\left(1-e^{2 \pi i z} q^{j}\right) \prod_{j=1}^{\infty}(1-$ $\left.e^{-2 \pi i z} q^{j}\right)$.

${ }^{8}$ The two cases $j=1,2$ require the Eisenstein summation prescription
}

$$
\sum_{m, n \in \mathbb{Z}} a_{m, n} \equiv \lim _{N \rightarrow \infty} \lim _{M \rightarrow \infty} \sum_{n=-N}^{N} \sum_{m=-M}^{M} a_{m, n}
$$




\subsubsection{Restoring double periodicity and modularity}

The quasi-periodicity of the Eisenstein-Kronecker series under $z \rightarrow z+\tau$ as given in eq. (3.13) can be lifted to an honest periodic behavior by defining

$$
\Omega(z, \alpha, \tau) \equiv \exp \left(2 \pi i \alpha \frac{\operatorname{Im}(z)}{\operatorname{Im}(\tau)}\right) F(z, \alpha, \tau) .
$$

Clearly, the resulting function $\Omega(z, \alpha, \tau)$ is doubly-periodic in $z$,

$$
\Omega(z+1, \alpha, \tau)=\Omega(z+\tau, \alpha, \tau)=\Omega(z, \alpha, \tau),
$$

and holomorphicity of the Eisenstein-Kronecker series eq. (3.11) gives rise to the differential equation

$$
\frac{\partial}{\partial \bar{z}} \Omega(z, \alpha, \tau)=-\frac{\pi \alpha}{\operatorname{Im}(\tau)} \Omega(z, \alpha, \tau) .
$$

The latter implies that the connection $J$ in eq. (3.4) satisfies the integrability condition eq. (3.3) and generates homotopy-invariant iterated integrals via the formal power series eq. (3.5) [16].

Upon taking the exponential in eq. (3.15) into account, the modular transformation properties of the Eisenstein-Kronecker series [52, 55], can be translated into

$$
\Omega\left(\frac{z}{c \tau+d}, \frac{\alpha}{c \tau+d}, \frac{a \tau+b}{c \tau+d}\right)=(c \tau+d) \Omega(z, \alpha, \tau)
$$

for $\left(\begin{array}{ll}a & b \\ c & d\end{array}\right) \in \mathrm{SL}(2, \mathbb{Z})$. The Fay identity eq. (3.14) for the Eisenstein-Kronecker series carries over to

$$
\begin{aligned}
\Omega\left(z_{1}, \alpha_{1}, \tau\right) \Omega\left(z_{2}, \alpha_{2}, \tau\right)= & \Omega\left(z_{1}, \alpha_{1}+\alpha_{2}, \tau\right) \Omega\left(z_{2}-z_{1}, \alpha_{2}, \tau\right) \\
& +\Omega\left(z_{2}, \alpha_{1}+\alpha_{2}, \tau\right) \Omega\left(z_{1}-z_{2}, \alpha_{1}, \tau\right)
\end{aligned}
$$

after multiplication with $\exp \left(\frac{2 \pi i}{\operatorname{Im}(\tau)}\left[\alpha_{1} \operatorname{Im}\left(z_{1}\right)+\alpha_{2} \operatorname{Im}\left(z_{2}\right)\right]\right)$.

\subsection{Definition and properties of the weighting functions $f^{(n)}$}

\subsubsection{Definition of $f^{(n)}$}

We define the functions $f^{(n)}$ entering the eIIs eq. (2.18) through the following Taylor series in $\alpha$,

$$
\alpha \Omega(z, \alpha, \tau) \equiv \sum_{n=0}^{\infty} f^{(n)}(z, \tau) \alpha^{n} .
$$

They are real analytic on the punctured elliptic curve $E_{\tau}^{\times}$. As above, we will omit the argument $\tau$ and write $f^{(n)}(z)$ or often simply $f^{(n)}$. Their explicit form is conveniently captured by the following functions ${ }^{9} \mathcal{E}_{n}$

$$
\mathcal{E}_{1}(z, \tau) \equiv E_{1}(z, \tau)+2 \pi i \frac{\operatorname{Im}(z)}{\operatorname{Im}(\tau)}, \quad \mathcal{E}_{n}(z, \tau) \equiv(-1)^{n}\left(e_{n}(\tau)-E_{n}(z, \tau)\right) \forall n \geq 2 .
$$

\footnotetext{
${ }^{9}$ Note that all $\mathcal{E}_{n}$ are meromorphic except for $\mathcal{E}_{1}$ (due to the term $\operatorname{Im}(z)$ ), and that $\mathcal{E}_{2}(z)=-\wp(z)$ is the Weierstrass function. Higher functions $\mathcal{E}_{n}$ at $n \geq 3$ are related to derivatives of the Weierstrass function, e.g. $\mathcal{E}_{3}=-\frac{1}{2} \partial \wp$ and $\mathcal{E}_{4}=e_{4}-\frac{1}{6} \partial^{2} \wp$.
} 
These functions result in a simple representation of the generating series

$$
\alpha \Omega(z, \alpha, \tau)=\exp \left(\sum_{j=1}^{\infty} \frac{\alpha^{j}}{j} \mathcal{E}_{j}(z, \tau)\right)
$$

and allow for a combinatorial interpretation of $f^{(n)}(z, \tau)$ in terms of the cycle index of the symmetric group $S_{n}$ (see appendix D).

Comparison with eq. (3.20) yields the following expressions for the lowest functions $f^{(n)}$

$$
\begin{aligned}
f^{(1)} & =\mathcal{E}_{1} \\
f^{(2)} & =\frac{1}{2}\left(\mathcal{E}_{1}^{2}+\mathcal{E}_{2}\right) \\
f^{(3)} & =\frac{1}{3 !}\left(\mathcal{E}_{1}^{3}+3 \mathcal{E}_{1} \mathcal{E}_{2}+2 \mathcal{E}_{3}\right) \\
f^{(4)} & =\frac{1}{4 !}\left(\mathcal{E}_{1}^{4}+6 \mathcal{E}_{1}^{2} \mathcal{E}_{2}+8 \mathcal{E}_{1} \mathcal{E}_{3}+3 \mathcal{E}_{2}^{2}+6 \mathcal{E}_{4}\right) \\
f^{(5)} & =\frac{1}{5 !}\left(\mathcal{E}_{1}^{5}+10 \mathcal{E}_{1}^{3} \mathcal{E}_{2}+20 \mathcal{E}_{1}^{2} \mathcal{E}_{3}+15 \mathcal{E}_{1} \mathcal{E}_{2}^{2}+30 \mathcal{E}_{1} \mathcal{E}_{4}+20 \mathcal{E}_{2} \mathcal{E}_{3}+24 \mathcal{E}_{5}\right) .
\end{aligned}
$$

The functions $\mathcal{E}_{j}$ can be expressed in terms of $\ln \theta_{1}$ via eq. (3.10), which leads to the representations for $f^{(1)}$ and $f^{(2)}$ provided in eqs. (2.15) and (2.16). As shown in appendix D, the general expression for $f^{(n)}$ following from eq. (3.22) reads

$$
f^{(n)}=\sum_{a_{1}, a_{2}, \ldots, a_{n} \geq 0} \delta\left(\sum_{i=1}^{n} i a_{i}-n\right) \prod_{j=1}^{n} \frac{\mathcal{E}_{j}^{a_{j}}}{j^{a_{j}} a_{j} !},
$$

and an equivalent recursive representation is given by

$$
f^{(n)}=\frac{1}{n} \sum_{j=1}^{n} \mathcal{E}_{j} f^{(n-j)} .
$$

\subsubsection{Properties of $f^{(n)}$}

The functions $f^{(n)}$ inherit their double periodicity, the form of their antiholomorphic derivative as well as their behavior under modular transformations from the generating series in eqs. (3.16), (3.17) and (3.18):

$$
\begin{aligned}
f^{(n)}(z+1) & =f^{(n)}(z+\tau)=f^{(n)}(z) \\
\frac{\partial f^{(n)}(z)}{\partial \bar{z}} & =-\frac{\pi}{\operatorname{Im}(\tau)} f^{(n-1)}(z) \\
f^{(n)}\left(\frac{z}{c \tau+d}, \frac{a \tau+b}{c \tau+d}\right) & =(c \tau+d)^{n} f^{(n)}(z, \tau) .
\end{aligned}
$$

Likewise, the Fay identity eq. (3.19) implies for $f_{i j}^{(n)} \equiv f^{(n)}\left(z_{i}-z_{j}\right)$ :

$$
f_{i l}^{(m-1)} f_{j l}^{(n)}+f_{i l}^{(m)} f_{j l}^{(n-1)}=\sum_{r=0}^{n}\left(\begin{array}{c}
m-1+r \\
r
\end{array}\right) f_{j i}^{(n-r)} f_{i l}^{(m-1+r)}+\sum_{r=0}^{m}\left(\begin{array}{c}
n-1+r \\
r
\end{array}\right) f_{i j}^{(m-r)} f_{j l}^{(n-1+r)} .
$$


This identity has been used repeatedly to derive relations among eIIs in section 2 (cf. eq. (2.40) above).

Given the singular factor $\frac{\theta_{1}^{\prime}(0, \tau)}{\theta_{1}(z, \tau)}=\frac{1}{z}+\mathcal{O}(z)$ in the Eisenstein-Kronecker series eq. (3.11), one can check that the residue at the simple pole of $\Omega$ at the origin is independent on $\alpha$. Hence, only $f^{(1)}$ has a simple pole at any $z=k+\tau l$ for $k, l \in \mathbb{Z}$ whereas all other weighting functions $f^{(n \neq 1)}$ are regular on the entire elliptic curve:

$$
\lim _{z \rightarrow 0} z f^{(n)}(z)=\delta_{n, 1} .
$$

It is this property of the functions $f^{(n)}$, which is responsible for the $z \rightarrow 0$ behavior stated in eq. (2.29).

\subsection{3 q-expansions of $f^{(n)}$}

The Eisenstein-Kronecker series eq. (3.11) is known to have the following power-series expansion in $q=e^{2 \pi i \tau}[16,23]$

$$
\begin{aligned}
\alpha F(z, \alpha, \tau) & =1+\pi \alpha \cot (\pi z)-2 \sum_{k=1}^{\infty} \zeta_{2 k} \alpha^{2 k}-2 \pi i \alpha \sum_{m, n=1}^{\infty}\left(e^{2 \pi i(m z+n \alpha)}-e^{-2 \pi i(m z+n \alpha)}\right) q^{m n} \\
& \equiv \sum_{n=0}^{\infty} g^{(n)}(z) \alpha^{n} .
\end{aligned}
$$

Disentangling the powers of $\alpha$ yields the holomorphic parts $g^{(n)}$ of the functions $f^{(n)}$, e.g.

$$
\begin{aligned}
& g^{(1)}(z)=\pi \cot (\pi z)+4 \pi \sum_{m=1}^{\infty} \sin (2 \pi m z) \sum_{n=1}^{\infty} q^{m n} \\
& g^{(2)}(z)=-2 \zeta_{2}+8 \pi^{2} \sum_{m=1}^{\infty} \cos (2 \pi m z) \sum_{n=1}^{\infty} n q^{m n} \\
& g^{(3)}(z)=-8 \pi^{3} \sum_{m=1}^{\infty} \sin (2 \pi m z) \sum_{n=1}^{\infty} n^{2} q^{m n}
\end{aligned}
$$

where $\cot (\pi z)=\frac{1}{\pi z}+\mathcal{O}(z)$ captures the simple pole of $f^{(1)}$. More generally, we find

$$
\begin{aligned}
\left.g^{(k)}(z)\right|_{k=2,4, \ldots} & =-2\left[\zeta_{k}+\frac{(2 \pi i)^{k}}{(k-1) !} \sum_{m=1}^{\infty} \cos (2 \pi m z) \sum_{n=1}^{\infty} n^{k-1} q^{m n}\right] \\
\left.g^{(k)}(z)\right|_{k=3,5, \ldots} & =-2 i \frac{(2 \pi i)^{k}}{(k-1) !} \sum_{m=1}^{\infty} \sin (2 \pi m z) \sum_{n=1}^{\infty} n^{k-1} q^{m n} .
\end{aligned}
$$

The non-holomorphic piece in $f^{(n)}$ consisting of factors $\frac{\operatorname{Im}(z)}{\operatorname{Im}(\tau)}$ can be immediately restored via

$$
f^{(n)}(z)=\sum_{k=0}^{n} \frac{[2 \pi i \operatorname{Im}(z)]^{k}}{k ![\operatorname{Im}(\tau)]^{k}} g^{(n-k)}(z) .
$$

Even though the functions $f^{(n)}$ in the definition eq. (2.18) of eIIs are evaluated at real arguments in the subsequent, we will keep track of the admixtures of $\operatorname{Im}(z)$ in eq. (3.37) 
for further applications beyond this work. For example, another system of eIIs and eMZVs can be defined for the path from 0 to $\tau$ instead of the real interval $[0,1]$ whose properties are crucially affected by the factors of $\operatorname{Im}(z)$ and the resulting modular properties.

\section{The one-loop four-point amplitude in open string theory}

Iterated integrals defined on an elliptic curve in subsection 2.2 appear naturally in superstring theory. Calculating one-loop scattering amplitudes among open string states amounts to evaluating iterated integrals weighted by the functions $f^{(n)}$ defined in section 3. Accordingly, the expansion of one-loop superstring amplitudes in the inverse string tension $\alpha^{\prime}$ involves eMZVs.

The $\alpha^{\prime}$-expansion of tree-level amplitudes in open string theory is well known to involve standard MZVs, see e.g. ref. [34]. The pattern of their appearance is much simpler as compared to the MZVs and polylogarithms in loop amplitudes of field theory and can be understood in terms of motivic MZVs [29] as well as the Drinfeld associator [38]. Hence, it is not surprising that one-loop string amplitudes furnish a perfect laboratory to study patterns and properties of eMZVs.

Iterated integrals in one-loop open string amplitudes occur on the boundaries of a two-dimensional worldsheet of either cylinder or Möbius-strip topology [40]. They describe conformally inequivalent configurations of inserting open string states on the respective boundaries. As a first field of application for eMZVs, we will entirely focus on cylindrical worldsheets in this work with all integrations confined to one boundary. ${ }^{10}$ As shown in figure 1 , this situation can be described by a torus with purely imaginary modular parameter $\tau=i t$ with $t \in \mathbb{R}$. The cylinder boundaries are then parametrized by $\operatorname{Re}\left(z_{j}\right) \in[0,1]$ with $\operatorname{Im}\left(z_{j}\right)=0$ and $\operatorname{Im}\left(z_{j}\right)=\frac{t}{2}$, respectively. The configuration of interest with one boundary empty is captured by real insertion points $z_{j} \in \mathbb{R}$.

\subsection{The four-point amplitude}

For massless open-string excitations in ten dimensions - gluons and gluinos - supersymmetry requires at least four external states for a non-vanishing one-loop amplitude, so the simplest case to be studied below is the four-point function [59, 60],

$$
\begin{aligned}
A_{\text {string }}^{1-\text { loop }}(1,2,3,4) & =s_{12} s_{23} A_{\mathrm{YM}}^{\text {tree }}(1,2,3,4) \int_{0}^{\infty} \mathrm{d} t I_{4 \mathrm{pt}}(1,2,3,4) \\
I_{4 \mathrm{pt}}(1,2,3,4) & \equiv \int_{0}^{1} \mathrm{~d} z_{4} \int_{0}^{z_{4}} \mathrm{~d} z_{3} \int_{0}^{z_{3}} \mathrm{~d} z_{2} \int_{0}^{z_{2}} \mathrm{~d} z_{1} \delta\left(z_{1}\right) \prod_{j<k}^{4} \exp \left[s_{j k} P_{j k}\right] .
\end{aligned}
$$

The entire polarization dependence is captured by the four-point tree amplitude of sYM field theory, see [61] for its tensor structure. The worldsheet integral $I_{4 \mathrm{pt}}(1,2,3,4)$ depends on the external momenta $k_{i}$ through dimensionless Mandelstam invariants

$$
s_{i j} \equiv \alpha^{\prime}\left(k_{i}+k_{j}\right)^{2},
$$

\footnotetext{
${ }^{10}$ The interplay between open string worldsheets of different topologies is crucial for the cancellations of infinities [56] and anomalies [57, 58] which occur for gauge group $\mathrm{SO}(32)$.
} 


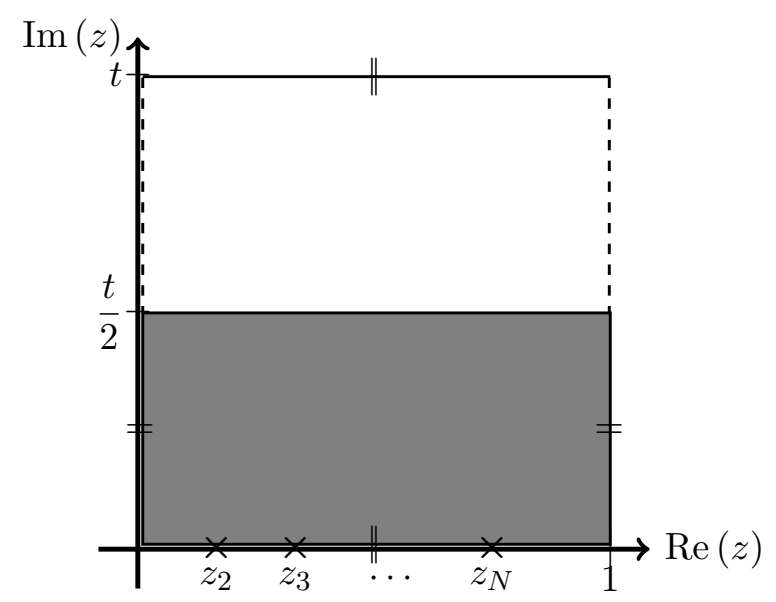

Figure 1. Parametrization of the cylinder worldsheet through the shaded region. The boundary under investigation has real coordinates $z_{j} \in[0,1]$. The identified edges inherited from the underlying torus at $\tau=i t$ are marked by $=$ and $\|$, respectively.

where momentum conservation and the mass-shell condition $k_{i}^{2}=0$ leave two independent $s_{i j}$,

$$
s_{34}=s_{12}, \quad s_{14}=s_{23}, \quad s_{13}=s_{24}=-s_{12}-s_{23} .
$$

The dependence on worldsheet positions $z_{j} \in[0,1]$ enters through the genus-one Green function

$$
P_{i j} \equiv \ln \left|\frac{\theta_{1}\left(z_{i}-z_{j}, \tau\right)}{\theta_{1}^{\prime}(0, \tau)}\right|^{2}-\frac{2 \pi}{\operatorname{Im}(\tau)}\left[\operatorname{Im}\left(z_{i}-z_{j}\right)\right]^{2}
$$

which is related to the singular function $f_{i j}^{(1)} \equiv f^{(1)}\left(z_{i}-z_{j}\right)$ in eq. (2.15) via

$$
\partial P_{i j}=f_{i j}^{(1)}, \quad P_{i j}=\int_{z_{j}}^{z_{i}} \mathrm{~d} w f^{(1)}\left(w-z_{j}\right) .
$$

The endpoint divergence as $w \rightarrow z_{j}$ can be dealt with through the regularization prescription eq. (2.26) which heuristically amounts to $\lim _{z_{i} \rightarrow z_{j}} P_{i j}=0$. Note that the dependence of $I_{4 \mathrm{pt}}(1,2,3,4)$ on $s_{i j}$ and $q \equiv e^{-2 \pi t}$ is suppressed for ease of notation.

The non-holomorphic piece in $f^{(1)}(z) \equiv \partial \ln \theta_{1}(z, \tau)+2 \pi i \frac{\operatorname{Im} z}{\operatorname{Im} \tau}$ drops out for the present cylinder parametrization where all vertices are inserted on the boundary with real coordinates $z_{j}$. Accordingly, the differential form $\nu \sim \mathrm{d} \operatorname{Im}(z)$ in eq. (3.1) required for homotopy invariance does not contribute to the cylinder integrals under consideration. However, the admixtures of $\frac{\operatorname{Im} z}{\operatorname{Im} \tau}$ in $f^{(n)}$ are crucial for modular invariance of closed-string amplitudes and cylinder diagrams with open string states on both boundaries.

Translation invariance on genus-one surfaces can be used to fix $z_{1}=0$. In addition, the $N$-point integration measure which appears for $N=4$ in eq. (4.2),

$$
\int_{12 \ldots N} \equiv \int_{0}^{1} \mathrm{~d} z_{N} \int_{0}^{z_{N}} \mathrm{~d} z_{N-1} \ldots \int_{0}^{z_{3}} \mathrm{~d} z_{2} \int_{0}^{z_{2}} \mathrm{~d} z_{1} \delta\left(z_{1}\right)
$$


is invariant under cyclic shifts $z_{i} \rightarrow z_{i+1 \bmod N}$ and, up to a sign $(-1)^{N}$, under reflection $z_{i} \rightarrow z_{N+1-i}$. Some features of the one-loop $N$-point amplitudes are discussed in section 5 . Their integrand then involves factors of $f^{\left(w_{i}\right)}\left(z_{j}-z_{k}\right)$ with overall weight $\sum_{i} w_{i}=N-4$.

As another generalization of the one-loop amplitude eq. (4.1) in ten spacetime dimensions, one could consider supersymmetry-preserving compactifications on a torus. For each circular dimension of radius $R$, the associated momentum components are quantized and contribute a correction factor of $\sum_{n=-\infty}^{\infty} e^{-n^{2} \pi t R^{2} / \alpha^{\prime}}$ to the $t$-integrand [62]. Since this does not affect the $z_{j}$-integrations within $I_{4 \mathrm{pt}}(1,2,3,4)$ and the resulting eMZVs, the subsequent results on the $\alpha^{\prime}$-expansion are universal for any torus compactification to spacetime dimensions $D \leq 10$.

\subsection{The $\alpha^{\prime}$-expansion}

In this section, we investigate the $\alpha^{\prime}$-expansion of the $t$-integrand in eq. (4.1),

$$
I_{4 \mathrm{pt}}(1,2,3,4)=\int_{1234} \prod_{i<j}^{4} \sum_{n_{i j}=0}^{\infty} \frac{1}{n_{i j} !}\left(s_{i j} P_{i j}\right)^{n_{i j}},
$$

which encodes the low-energy effective action for the gluon supermultiplet. Expanding in $\alpha^{\prime}$ amounts to Taylor expanding the exponential in eq. (4.2) in all the Mandelstam invariants $s_{i j}$ defined in eq. (4.3) as well as the corresponding worldsheet Green function $P_{i j}$ given by eq. (4.6).

In addition to the power-series expansion in $\alpha^{\prime}$ discussed in the subsequent, the integration region of large $t$ in the amplitude eq. (4.1) gives rise to logarithmic, non-analytic momentum dependence. The associated threshold singularities in $s_{i j}$ are for instance crucial to make contact with the Feynman box integral in the sYM amplitude arising in the point-particle limit [62]. Mimicking the low energy-analysis of closed string one-loop amplitudes [63-66], we separate the analytic from the non-analytic parts of the amplitude and do not keep track of the non-analytic threshold singularities.

The simplest monomials in $P_{i j}$ inequivalent under cyclic shifts and reflections of the vertex positions $z_{j}$ integrate to

$$
c_{0} \equiv \int_{1234} 1, \quad c_{1}^{1} \equiv \int_{1234} P_{12}, \quad c_{2}^{1} \equiv \int_{1234} P_{13} .
$$

At second and third order in $\alpha^{\prime}$ one finds

$$
\begin{array}{rlrl}
c_{1}^{2} & \equiv \frac{1}{2} \int_{1234} P_{12}^{2}, & c_{3}^{2} \equiv \int_{1234} P_{12} P_{14}, & c_{5}^{2} \equiv \int_{1234} P_{12} P_{34} \\
c_{2}^{2} \equiv \frac{1}{2} \int_{1234} P_{13}^{2}, & c_{4}^{2} \equiv \int_{1234} P_{13} P_{24}, & c_{6}^{2} \equiv \int_{1234} P_{12} P_{13}
\end{array}
$$

as well as

$$
\begin{aligned}
& c_{1}^{3} \equiv \frac{1}{6} \int_{1234} P_{12}^{3}, \quad c_{5}^{3} \equiv \frac{1}{2} \int_{1234} P_{12}^{2} P_{34}, \quad c_{9}^{3} \equiv \int_{1234} P_{12} P_{13} P_{23} \\
& c_{2}^{3} \equiv \frac{1}{6} \int_{1234} P_{13}^{3}, \quad c_{6}^{3} \equiv \frac{1}{2} \int_{1234} P_{12}^{2} P_{13}, \quad c_{10}^{3} \equiv \int_{1234} P_{12} P_{13} P_{14}
\end{aligned}
$$




$$
\begin{array}{rlrl}
c_{3}^{3} & \equiv \frac{1}{2} \int_{1234} P_{12}^{2} P_{23}, & c_{7}^{3} \equiv \frac{1}{2} \int_{1234} P_{12} P_{13}^{2}, & c_{11}^{3} \equiv \int_{1234} P_{12} P_{34} P_{13} \\
c_{4}^{3} \equiv \frac{1}{2} \int_{1234} P_{13}^{2} P_{24}, & c_{8}^{3} \equiv \int_{1234} P_{12} P_{23} P_{34}, & c_{12}^{3} \equiv \int_{1234} P_{13} P_{24} P_{12} .
\end{array}
$$

As will be demonstrated in section 4.3, eMZVs defined in eq. (2.22) are the natural language to describe the above $c_{i}^{j}$ and to understand the linear combinations appearing after applying momentum conservation eq. (4.4):

$$
\begin{aligned}
& I_{4 \mathrm{pt}}(1,2,3,4)=c_{0}+2\left(c_{1}^{1}-c_{2}^{1}\right)\left(s_{12}+s_{23}\right)+\left(2 c_{1}^{2}+2 c_{2}^{2}-c_{3}^{2}-c_{4}^{2}\right)\left(s_{12}^{2}+\frac{1}{4} s_{12} s_{23}+s_{23}^{2}\right) \\
& +\frac{1}{4}\left(-2 c_{1}^{2}+14 c_{2}^{2}+c_{3}^{2}-7 c_{4}^{2}\right) s_{12} s_{23}+2\left(c_{10}^{3}-2 c_{1}^{3}-c_{2}^{3}+2 c_{3}^{3}+c_{4}^{3}-2 c_{9}^{3}\right) s_{12} s_{23}\left(s_{12}+s_{23}\right) \\
& +\left(2 c_{10}^{3}+2 c_{1}^{3}-2 c_{2}^{3}+6 c_{3}^{3}+2 c_{4}^{3}-8 c_{6}^{3}-2 c_{8}^{3}\right)\left(s_{12}+s_{23}\right)\left(s_{12}^{2}+s_{12} s_{23}+s_{23}^{2}\right)+\mathcal{O}\left(\alpha^{\prime 4}\right) .
\end{aligned}
$$

A first flavor of relations among $c_{i}^{j}$ (and thus ultimately among eMZVs) can be obtained by exploiting cyclic and reflection properties of five-point integrals such as

$$
\int_{12345} P_{45} \partial_{2} P_{23}=\int_{12345} P_{51} \partial_{2} P_{23} \Rightarrow \int_{1345} P_{45} P_{13}=\int_{1345} P_{51} P_{13} \Rightarrow c_{3}^{2}=c_{5}^{2},
$$

see eq. (4.7) for the measure $\int_{12345}$. Similar methods imply that

$$
2 c_{6}^{2}=c_{3}^{2}+c_{4}^{2}, \quad c_{3}^{3}=c_{5}^{3}, \quad c_{10}^{3}=c_{11}^{3}, \quad c_{7}^{3}+c_{6}^{3}=c_{3}^{3}+c_{4}^{3}, \quad c_{11}^{3}+c_{10}^{3}=c_{8}^{3}+c_{12}^{3},
$$

these relations have been used to eliminate $c_{5}^{2}, c_{6}^{2}$ as well as $c_{5}^{3}, c_{7}^{3}, c_{12}^{3}, c_{11}^{3}$ from eq. (4.12).

Note that the $\alpha^{\prime}$-expansion of closed string one-loop amplitudes has been analyzed along similar lines in refs. [63-66]. Since each closed-string insertion point $z_{j}$ is integrated over the entire torus $E_{\tau}$, integrals involving propagators with a free endpoint vanish and therefore much fewer closed-string counterparts of the coefficients $c_{i}^{j}$ arise.

\subsection{Elliptic multiple zeta values}

In this section we convert the constituents of the $\alpha^{\prime}$-expansion, $c_{i}^{j}$ defined by eqs. (4.9), (4.10) and (4.11), to eMZVs. This will provide a characterization of the particular linear combinations of $c_{i}^{j}$ which appear in eq. (4.12) along with various powers of $s_{12}$ and $s_{23}$.

The leading term $c_{0}$ in eq. (4.9) can be straightforwardly evaluated to yield $\frac{1}{6}$ and furnishes a special case of

$$
\omega(\underbrace{0,0, \ldots, 0}_{n})=\frac{1}{n !},
$$

which follows from multiple insertions of $1=f^{(0)}\left(z_{i}\right)$. Nevertheless, it will prove instructive for the comparison with higher orders in $\alpha^{\prime}$ to express $c_{0}$ as an unevaluated eMZV:

$$
\begin{aligned}
c_{0} & =\int_{0}^{1} f^{(0)}\left(z_{4}\right) \mathrm{d} z_{4} \int_{0}^{z_{4}} f^{(0)}\left(z_{3}\right) \mathrm{d} z_{3} \int_{0}^{z_{3}} f^{(0)}\left(z_{2}\right) \mathrm{d} z_{2} \\
& =\Gamma(0,0,0 ; 1)=\omega(0,0,0) .
\end{aligned}
$$

Below, we will repeatedly apply the definitions eq. (2.18) and eq. (2.22) of eIIs and eMZVs, respectively, in order to express the other integrals $c_{i}^{j}$ in the same fashion. 


\subsubsection{First order in $P_{i j}$ : integrals $c_{i}^{1}$}

At linear order in $s_{i j}$, we substitute $P_{1 j}=\int_{0}^{z_{j}} f^{(1)}(w) \mathrm{d} w$ according to eq. (4.6) and $z_{1}=0$ into the definitions eq. (4.9) and find

$$
\begin{aligned}
c_{1}^{1} & =\int_{0}^{1} f^{(0)}\left(z_{4}\right) \mathrm{d} z_{4} \int_{0}^{z_{4}} f^{(0)}\left(z_{3}\right) \mathrm{d} z_{3} \int_{0}^{z_{3}} f^{(0)}\left(z_{2}\right) \mathrm{d} z_{2} \int_{0}^{z_{2}} f^{(1)}(w) \mathrm{d} w \\
& =\Gamma(0,0,0,1 ; 1)=\omega(1,0,0,0) \\
c_{2}^{1} & =\int_{0}^{1} f^{(0)}\left(z_{4}\right) \mathrm{d} z_{4} \int_{0}^{z_{4}} f^{(0)}\left(z_{3}\right) \mathrm{d} z_{3} \int_{0}^{z_{3}} f^{(0)}\left(z_{2}\right) \mathrm{d} z_{2} \int_{0}^{z_{3}} f^{(1)}(w) \mathrm{d} w \\
& =\int_{0}^{1} f^{(0)}\left(z_{4}\right) \mathrm{d} z_{4} \int_{0}^{z_{4}} f^{(0)}\left(z_{3}\right) \mathrm{d} z_{3} \Gamma\left(0 ; z_{3}\right) \Gamma\left(1 ; z_{3}\right) \\
& =\int_{0}^{1} f^{(0)}\left(z_{4}\right) \mathrm{d} z_{4} \int_{0}^{z_{4}} f^{(0)}\left(z_{3}\right) \mathrm{d} z_{3}\left[\Gamma\left(1,0 ; z_{3}\right)+\Gamma\left(0,1 ; z_{3}\right)\right] \\
& =\Gamma(0,0,0,1 ; 1)+\Gamma(0,0,1,0 ; 1)=\omega(1,0,0,0)+\omega(0,1,0,0) .
\end{aligned}
$$

The second line of eq. (4.18) makes use of the shuffle product eq. (2.19) for eIIs. Equivalence of eq. (4.17) with the cyclically shifted integrand

$$
\begin{aligned}
\int_{1234} P_{14} & =\int_{0}^{1} f^{(0)}\left(z_{4}\right) \mathrm{d} z_{4} \int_{0}^{z_{4}} f^{(0)}\left(z_{3}\right) \mathrm{d} z_{3} \int_{0}^{z_{3}} f^{(0)}\left(z_{2}\right) \mathrm{d} z_{2} \int_{0}^{z_{4}} f^{(1)}(w) \mathrm{d} w \\
& =\omega(1,0,0,0)+\omega(0,1,0,0)+\omega(0,0,1,0)
\end{aligned}
$$

can be checked using antisymmetry $\omega(0,1,0,0)+\omega(0,0,1,0)=0$ following from eq. (2.24).

\subsubsection{Second order in $P_{i j}$ : integrals $c_{i}^{2}$}

At quadratic order in $s_{i j}$, the rewriting $P_{1 j}=\int_{0}^{z_{j}} f^{(1)}(w) \mathrm{d} w=-\int_{z_{j}}^{1} f^{(1)}(w) \mathrm{d} w$ allows to straightforwardly address any quadratic monomial in $P_{12}, P_{13}, P_{14}$ along the lines of eqs. (4.17) and (4.18):

$$
\begin{aligned}
& c_{1}^{2}=\omega(1,1,0,0,0) \\
& c_{2}^{2}=\omega(1,1,0,0,0)+\omega(1,0,1,0,0)+\omega(0,1,1,0,0) \\
& c_{3}^{2}=-\omega(1,0,0,0,1) \\
& c_{6}^{2}=2 \omega(1,1,0,0,0)+\omega(1,0,1,0,0) .
\end{aligned}
$$

Then, eqs. (4.13) and (4.14) can be used to determine the remaining two $c_{j}^{2}$ in eq. (4.10):

$$
\begin{aligned}
& c_{4}^{2}=2 \omega(1,1,0,0,0)+\omega(1,0,1,0,0)-\omega(1,0,0,1,0) \\
& c_{5}^{2}=-\omega(1,0,0,0,1) .
\end{aligned}
$$

Note that the integration limits $\int_{0}^{z_{j}} \ldots$ in the representation of $P_{1 j}$ can be traded for $-\int_{z_{j}}^{1} \ldots$. This is equivalent to applying a shuffle relation eq. (2.23),

$$
\begin{aligned}
& 0=\omega(1) \omega(1,0,0,0)=2 \omega(1,1,0,0,0)+\omega(1,0,1,0,0)+\omega(1,0,0,1,0)+\omega(1,0,0,0,1) \\
& 0=\omega(1) \omega(0,1,0,0)=\omega(1,0,1,0,0)+2 \omega(0,1,1,0,0)+\omega(0,1,0,1,0)+\omega(0,1,0,0,1)
\end{aligned}
$$

where $\omega(1)$ vanishes by the reflection identity eq. (2.24). 


\subsubsection{Integration techniques for $P_{23}, P_{24}, P_{34}$}

Green functions $P_{i j}$ where both indices describe a leg to be integrated (legs 2,3,4) are more difficult to integrate. Their integral representation eq. (4.6) inevitably gives rise to iterated integrals $\Gamma\left(\begin{array}{ccc}n_{1} & \ldots & n_{r} \\ a_{1} & \ldots & a_{r}\end{array} ; z\right)$ with the argument appearing in the labels, that is $a_{i}=z$. Integration over $z_{3}$ and $z_{4}$ then requires the techniques of subsection 2.2.2, in particular the recursion formulæ eq. (2.41) to eq. (2.43).

The simple corollary $\Gamma\left(\begin{array}{cc}1 & 0 \\ z & 0\end{array} ;\right)=-\Gamma\left(\begin{array}{cc}0 & 1 \\ 0 & 0\end{array} ; z\right)$ of the reflection identity eq. $(2.20)$ is sufficient to integrate $P_{23}$ and to reproduce eq. (4.17) from a different cyclic representative. The quadratic case $c_{5}^{2}=\int_{1234} P_{12} P_{34}$, on the other hand, requires more effort. One obtains

$$
\begin{aligned}
\int_{1234} P_{12} P_{34} & =-\int_{0}^{1} f^{(0)} \mathrm{d} z_{4} \int_{0}^{z_{4}} f^{(1)}\left(w-z_{4}\right) \mathrm{d} w \int_{0}^{w} f^{(0)} \mathrm{d} z_{3} \int_{0}^{z_{3}} f^{(0)} \mathrm{d} z_{2} \int_{0}^{z_{2}} f^{(1)}(u) \mathrm{d} u \\
& =-\int_{0}^{1} f^{(0)}\left(z_{4}\right) \mathrm{d} z_{4} \Gamma\left(\begin{array}{cccc}
1 & 0 & 0 & 1 \\
z_{4} & 0 & 0 & 0
\end{array} ; z_{4}\right) \\
& =2 \omega(1,1,0,0,0)-2 \omega(2,0,0,0,0)-\omega(0,2,0,0,0)-\zeta_{2} \omega(0,0,0)
\end{aligned}
$$

where $\Gamma\left(\begin{array}{cccc}1 & 0 & 0 & 1 \\ z_{4} & 0 & 0 & 0\end{array} ; z_{4}\right)$ has been reexpressed via eq. $(2.49)$ in the last step. In order to reproduce the result of eq. (4.21b), $-\omega(1,0,0,0,1)$, one needs to combine the shuffle relations eqs. (4.22) and (4.23) with eqs. (2.58) and (2.59). The desired result then follows from the constant eMZVs in eq. (4.15) and $\omega(2)=-2 \zeta_{2}$ which is a special case of

$$
\omega(n)=\left\{\begin{array}{ccc}
-2 \zeta_{n} & : \quad n \text { even } \\
0 \quad & : \quad n \text { odd }
\end{array} .\right.
$$

The expression for $\omega(n)$ can be inferred from order $q^{0}$ in the expansions eqs. (3.35) and (3.36).

\subsubsection{Third order in $P_{i j}$ : integrals $c_{i}^{3}$}

Starting from the third order in Mandelstam variables, relations such as eq. (4.14) are no longer sufficient to reduce the complete list of $c_{i}^{3}$ in eq. (4.11) to elementary integrals over monomials in $P_{12}, P_{13}$ and $P_{14}$. Instead, the inevitable factors of $P_{23}, P_{24}$ and $P_{34}$ require the procedure described in eq. (4.24) together with the recursive identities eq. (2.41) to (2.43) in order to rearrange the labels of the eIIs. This allows to reduce integrals over arbitrary monomials in $P_{i j}$ with $1 \leq i<j \leq 4$ to eMZVs. The integrals $c_{i}^{3}$, which are cubic in $P_{i j}$, give rise to

$$
\begin{aligned}
c_{1}^{3} & =\omega(1,1,1,0,0,0) \\
c_{2}^{3} & =\omega(1,1,1,0,0,0)+\omega(1,1,0,1,0,0)+\omega(1,0,1,1,0,0)+\omega(0,1,1,1,0,0) \\
c_{3}^{3} & =-\omega(1,1,0,0,0,1) \\
c_{4}^{3} & =6 \omega(1,1,1,0,0,0)+3 \omega(1,1,0,1,0,0)+\omega(1,0,1,1,0,0)+\omega(1,1,0,0,0,1) \\
c_{5}^{3} & =-\omega(1,1,0,0,0,1) \\
c_{6}^{3} & =3 \omega(1,1,1,0,0,0)+\omega(1,1,0,1,0,0) \\
c_{7}^{3} & =3 \omega(1,1,1,0,0,0)+2 \omega(1,1,0,1,0,0)+\omega(1,0,1,1,0,0)
\end{aligned}
$$




$$
\begin{aligned}
c_{8}^{3}= & 2 \omega(2,0,0,0,0,1)+\omega(0,2,0,0,0,1)-2 \omega(1,1,0,0,0,1)-\zeta_{2} \omega(1,0,0,0) \\
c_{9}^{3}= & 2 \omega(2,0,0,0,1,0)+2 \omega(2,0,0,0,0,1)+\omega(0,2,0,0,1,0)+\omega(0,2,0,0,0,1) \\
& -2 \omega(1,1,0,0,1,0)-2 \omega(1,1,0,0,0,1)-\zeta_{2} \omega(1,0,0,0)-\zeta_{2} \omega(0,1,0,0) \\
c_{10}^{3}= & -2 \omega(1,1,0,0,0,1)-\omega(1,0,1,0,0,1) \\
c_{11}^{3}= & -2 \omega(1,1,0,0,0,1)-\omega(1,0,1,0,0,1) \\
c_{12}^{3}= & -2 \omega(2,0,0,0,0,1)-\omega(0,2,0,0,0,1)+\zeta_{2} \omega(1,0,0,0) \\
& -2 \omega(1,0,1,0,0,1)-2 \omega(1,1,0,0,0,1),
\end{aligned}
$$

where the occurrences of $\zeta_{2}$ can be traced back to eq. (2.47).

\subsubsection{Assembling the results}

Momentum conservation only admits particular linear combinations of $c_{i}^{j}$ in the four-point amplitude eq. (4.12). It turns out that for all cases considered divergent eMZVs with the singular integrand $f^{(1)}$ in the first or last position drop out. Up to third order in $s_{i j}$, we have

$$
\begin{aligned}
& I_{4 \mathrm{pt}}(1,2,3,4)=\omega(0,0,0)-2 \omega(0,1,0,0)\left(s_{12}+s_{23}\right)+2 \omega(0,1,1,0,0)\left(s_{12}^{2}+s_{23}^{2}\right) \\
& -2 \omega(0,1,0,1,0) s_{12} s_{23}+\beta_{5}\left(s_{12}^{3}+2 s_{12}^{2} s_{23}+2 s_{12} s_{23}^{2}+s_{23}^{3}\right)+\beta_{2,3} s_{12} s_{23}\left(s_{12}+s_{23}\right)+\mathcal{O}\left(\alpha^{\prime 4}\right)
\end{aligned}
$$

with

$$
\begin{aligned}
\beta_{5}= & \frac{4}{3}\left[\omega(0,0,1,0,0,2)+\omega(0,1,1,0,1,0)-\omega(2,0,1,0,0,0)-\zeta_{2} \omega(0,1,0,0)\right] \\
\beta_{2,3}= & \frac{1}{3} \omega(0,0,1,0,2,0)-\frac{3}{2} \omega(0,1,0,0,0,2)-\frac{1}{2} \omega(0,1,1,1,0,0) \\
& -2 \omega(2,0,1,0,0,0)-\frac{4}{3} \omega(0,0,1,0,0,2)-\frac{10}{3} \zeta_{2} \omega(0,1,0,0),
\end{aligned}
$$

and the pattern at higher orders is under investigation. The above expressions for $\beta_{5}$ and $\beta_{2,3}$ are obtained using various eMZV relations using the methods of subsection 2.2.3.

\subsection{On the $q$-expansion of eMZVs and the string amplitude}

The evaluation of eMZVs as initiated in eq. (4.15) and eq. (4.25) will be pursued systematically in $[49,67,68]$. In this section, we give a glimpse of non-trivial $q$-dependence in simple cases and provide consistency checks for the constant piece of the low energy expansion eq. (4.27) of the four-point amplitude.

\subsubsection{The simplest $q$-expansions}

To determine the $q$-expansions of the simplest eMZVs, we start from the expansions of $f^{(1)}$ and $f^{(2)}$ spelled out in eq. (3.37), which in turn is based on eqs. (3.32) and (3.33). Using the integrals in appendix $\mathrm{C}$, we arrive at

$$
\omega(0,1,0,0)=\frac{\zeta_{3}}{8 \zeta_{2}}+\frac{3}{2 \pi^{2}} \sum_{m, n=1}^{\infty} \frac{1}{m^{3}} q^{m n}
$$


as well as

$$
\begin{aligned}
& \omega(0,1,1,0,0)=\frac{\zeta_{2}}{15}-\frac{1}{2 \pi^{2}} \sum_{m, n=1}^{\infty} \frac{n}{m^{4}} q^{m n}+\frac{1}{3} \sum_{m, n=1}^{\infty} \frac{n}{m^{2}} q^{m n} \\
& \omega(0,1,0,1,0)=-\frac{\zeta_{2}}{60}+\frac{2}{\pi^{2}} \sum_{m, n=1}^{\infty} \frac{n}{m^{4}} q^{m n}-\frac{1}{3} \sum_{m, n=1}^{\infty} \frac{n}{m^{2}} q^{m n} .
\end{aligned}
$$

A systematic method is under investigation and will appear in [68]. Note that the $q$ dependence of all the examples above can be expressed in terms of the function $\mathrm{ELi}_{n, m}$ introduced in section 8 of ref. [11] at arguments $x=y=1$.

\subsubsection{The constant piece of eMZVs and the $\alpha^{\prime}$-derivative}

The $t$-integration in the four-point amplitude eq. (4.1) is divergent unless the choice of gauge group SO(32) leads to cancellations between the cylinder and the Möbius-strip diagram [56]. The divergence is interpreted as a zero-momentum dilaton propagating to the vacuum and therefore proportional to the derivative of the tree level amplitude with respect to $\alpha^{\prime}$ [59]. The latter is given by

$$
A_{\text {string }}^{\text {tree }}(1,2,3,4)=\frac{\Gamma\left(1+s_{12}\right) \Gamma\left(1+s_{23}\right)}{\Gamma\left(1+s_{12}+s_{23}\right)} A_{\mathrm{YM}}^{\text {tree }}(1,2,3,4)
$$

with $\alpha^{\prime}$-expansion

$$
\begin{aligned}
& \frac{\Gamma\left(1+s_{12}\right) \Gamma\left(1+s_{23}\right)}{\Gamma\left(1+s_{12}+s_{23}\right)}=\exp \left\{\sum_{k=2}^{\infty}(-1)^{k} \frac{\zeta_{k}}{k}\left[s_{12}^{k}+s_{23}^{k}-\left(s_{12}+s_{23}\right)^{k}\right]\right\} \\
& =1-\zeta_{2} s_{12} s_{23}+\zeta_{3} s_{12} s_{23}\left(s_{12}+s_{23}\right)-\zeta_{4} s_{12} s_{23}\left(s_{12}^{2}+\frac{1}{4} s_{12} s_{23}+s_{23}^{2}\right) \\
& \quad-\zeta_{5} s_{12} s_{23}\left(s_{12}^{3}+2 s_{12}^{2} s_{23}+2 s_{12} s_{23}^{2}+s_{23}^{3}\right)-\zeta_{2} \zeta_{3}\left(s_{12} s_{23}\right)^{2}\left(s_{12}+s_{23}\right)+\mathcal{O}\left(\alpha^{\prime 6}\right) .
\end{aligned}
$$

In the representation of the one-loop amplitude given in eq. (4.27), the divergence originates from the constant part of the eMZVs' power series expansion in $q=e^{2 \pi i \tau}=e^{-2 \pi t}$. A systematic method to extract the constant term of eMZVs will be described in ref. [49]. The resulting divergence in the above result is given by

$$
\begin{aligned}
\left.A_{\text {string }}^{1-\text { loop }}(1,2,3,4)\right|_{\text {div }}=\left.s_{12} s_{23} A_{\mathrm{YM}}^{\text {tree }}(1,2,3,4) I_{4 \mathrm{pt}}(1,2,3,4)\right|_{q^{0}} \\
=\frac{1}{2 \pi^{2}} s_{12} s_{23} A_{\mathrm{YM}}^{\text {tree }}(1,2,3,4)\left\{2 \zeta_{2}-3 \zeta_{3}\left(s_{12}+s_{23}\right)+4 \zeta_{4}\left(s_{12}^{2}+\frac{1}{4} s_{12} s_{23}+s_{23}^{2}\right)\right. \\
\left.\quad-5 \zeta_{5}\left(s_{12}^{3}+2 s_{12}^{2} s_{23}+2 s_{12} s_{23}^{2}+s_{23}^{3}\right)+5 \zeta_{2} \zeta_{3} s_{12} s_{23}\left(s_{12}+s_{23}\right)+\mathcal{O}\left(\alpha^{\prime 4}\right)\right\},
\end{aligned}
$$

which is consistent with the $\alpha^{\prime}$-derivative of the tree amplitude [59] upon comparison with eq. (4.34),

$$
\left.A_{\text {string }}^{1 \text {-loop }}(1,2,3,4)\right|_{\text {div }}=-\frac{\alpha^{\prime}}{2 \pi^{2}} \frac{\partial}{\partial \alpha^{\prime}} A_{\text {string }}^{\text {tree }}(1,2,3,4)
$$




\section{Multi-particle one-loop string amplitudes and $f^{(n)}$}

This section is devoted to one-loop amplitudes involving five and more open string states. We firstly provide the five-point extension of the four-point $\alpha^{\prime}$-expansion in eq. (4.27). It is secondly demonstrated that the doubly-periodic functions $f^{(n)}$ defined in section 3 naturally enter the calculation of one-loop amplitudes with any number of external legs.

\subsection{The five-point open string amplitude}

In the same way as the four-point open string amplitude in eq. (4.1) allows to factor out the polarization dependence via $A_{\mathrm{YM}}^{\text {tree }}(1,2,3,4)$, one can express the five-point string amplitude in a basis of color-ordered trees of YM theory [69]. BCJ relations [70] single out two independent subamplitudes $A_{\mathrm{YM}}^{\text {tree }}(1, \rho(2,3), 4,5)$ with permutation $\rho \in S_{2}$, and for convenience, we consider the same color orderings in the one-loop string theory counterparts:

$$
A_{\text {string }}^{1 \text {-loop }}(1, \sigma(2,3), 4,5)=\int_{0}^{\infty} \mathrm{d} t \sum_{\rho \in S_{2}} I_{5 \mathrm{pt}}(\sigma \mid \rho) A_{\mathrm{YM}}^{\mathrm{tree}}(1, \rho(2,3), 4,5) .
$$

The $2 \times 2$ matrix $I_{5 \mathrm{pt}}(\sigma \mid \rho)$ is the generalization of the four-point scalar integral $I_{4 \mathrm{pt}}(1,2,3,4)$. It can be assembled from the kinematic factors which were simplified in ref. [69] using the pure spinor formalism [71],

$$
\begin{aligned}
\sum_{\rho \in S_{2}} I_{5 \mathrm{pt}}(1 \mid \rho) A_{\mathrm{YM}}^{\text {tree }}(1, \rho(2,3), 4,5) & =\int_{12345} \prod_{k<l}^{5} \exp \left[s_{k l} P_{k l}\right] \\
& \times\left[s_{23} f_{23}^{(1)}\left\langle C_{1 \mid 23,4,5}\right\rangle+(23 \leftrightarrow 24,25,34,35,45)\right] \\
\left\langle C_{1 \mid 23,4,5}\right\rangle & =s_{45}\left(s_{24} A_{\mathrm{YM}}^{\text {tree }}(1,3,2,4,5)-s_{34} A_{\mathrm{YM}}^{\text {tree }}(1,2,3,4,5)\right) .
\end{aligned}
$$

The integration measure $\int_{12345}$ is defined in eq. (4.7), the functions $f_{i j}^{(1)}=f^{(1)}\left(z_{i}-z_{j}\right)$ stem from OPE contractions among the worldsheet fields and the five-point Mandelstam invariants eq. (4.3) can be cast into a five-dimensional basis via momentum conservation, e.g. $s_{13}=s_{45}-s_{12}-s_{23}$.

From the mathematical point of view, the only novel five-point ingredient as compared to the four-point amplitude is the extra factor of $f_{i j}^{(1)}=\partial P_{i j}$ in the integrand of eq. (5.2). Thanks to the embedding of $f^{(1)}$ into the framework of eIIs eq. (2.18), the $\alpha^{\prime}$-expansion of the integrals $\int_{12345} f_{i j}^{(1)} \prod_{k<l}^{5} \exp \left[s_{k l} P_{k l}\right]$ in eq. (5.3) is again captured by eMZVs. The detailed discussion of kinematic poles as well as the order-by-order treatment of the exponential will be discussed elsewhere; here we simply quote the final result:

$$
\begin{aligned}
I_{5 \mathrm{pt}}(\sigma \mid \rho)=[- & \omega(0,0,0) P_{2}-2 \omega(0,1,0,0) M_{3}-5 \omega(0,1,1,0,0) P_{4} \\
& \left.-\left(2 \omega(0,1,0,1,0)+\frac{1}{2} \omega(0,1,1,0,0)\right) L_{4}+\mathcal{O}\left(\alpha^{\prime 5}\right)\right]_{\sigma, \rho} .
\end{aligned}
$$

Up to weight two at order $\mathcal{O}\left(\alpha^{4}\right)$, the eMZV content is the same as in the four-point expansion eq. (4.27). The accompanying $2 \times 2$ matrices $P_{i}, M_{i}, L_{i}$ are indexed by permutations $\rho, \sigma$, and their entries are polynomials of degree $i$ in Mandelstam variables. The representatives $P_{i}$ and $M_{i}$ already appear in the $\alpha^{\prime}$-expansion of open-string tree amplitudes, along 
with even and odd Riemann zeta values $\zeta_{i}$, respectively [29]. Given that the low-energy limit of one-loop amplitudes at any multiplicity has the mass dimension of $s_{i j}^{2} A_{\mathrm{YM}}^{\text {tree }}(\ldots)$, the eMZV coefficients of $P_{i}, M_{i}, L_{i}$ have weight $i-2$. This amounts to a shift of -2 in weight in comparison to the MZV coefficients of $P_{i}, M_{i}$ at tree-level.

They are available at the website [39] whereas $L_{4}$ reads

$$
\begin{gathered}
\left(L_{4}\right)_{11}=s_{12}^{2} s_{23}^{2}+2 s_{12}^{2} s_{23} s_{24}+s_{12}^{2} s_{24}^{2}+2 s_{12}^{2} s_{23} s_{34}+2 s_{12} s_{13} s_{23} s_{34}+2 s_{12} s_{23}^{2} s_{34} \\
+2 s_{12}^{2} s_{24} s_{34}+s_{12} s_{13} s_{24} s_{34}+2 s_{12} s_{23} s_{24} s_{34}+s_{12}^{2} s_{34}^{2}+2 s_{12} s_{13} s_{34}^{2} \\
+s_{13}^{2} s_{34}^{2}+2 s_{12} s_{23} s_{34}^{2}+2 s_{13} s_{23} s_{34}^{2}+s_{23}^{2} s_{34}^{2} \\
\left(L_{4}\right)_{12}=-s_{13} s_{24}\left(3 s_{12} s_{23}+s_{13} s_{23}+s_{23}^{2}+2 s_{12} s_{24}+s_{13} s_{24}+s_{23} s_{24}\right. \\
\left.+3 s_{12} s_{34}+2 s_{13} s_{34}+3 s_{23} s_{34}\right)
\end{gathered}
$$

and $\left(L_{4}\right)_{22}=\left.\left(L_{4}\right)_{11}\right|_{2 \leftrightarrow 3}$ and $\left(L_{4}\right)_{21}=\left.\left(L_{4}\right)_{12}\right|_{2 \leftrightarrow 3}$. The relabelling $2 \leftrightarrow 3$ refers to the $i, j$ along with the Mandelstam invariants $s_{i j}$.

The four-point one-loop amplitude eq. (4.27) can be cast into the same form as eq. (5.4) upon setting $L_{4} \rightarrow 0$ and

$$
P_{2} \rightarrow-s_{12} s_{23}, \quad M_{3} \rightarrow s_{12} s_{23}\left(s_{12}+s_{23}\right), \quad P_{4} \rightarrow-\frac{2}{5} s_{12} s_{23}\left(s_{12}^{2}+\frac{1}{4} s_{12} s_{23}+s_{23}^{2}\right)
$$

in agreement with the four-point open string tree eq. (4.33). The pattern of eMZVs at higher orders in $\alpha^{\prime}$ as well as the properties of the novel matrices $L_{i}$ are left for further projects.

\subsection{Functions $f^{(n)}$ from the RNS formalism}

In this subsection we will show that the doubly-periodic functions $f^{(n)}$ for any $n$ are naturally generated in the one-loop amplitude computation using the RNS formalism [7274]. Their emergence in the parity-even and parity-odd sectors turns out to follow two separate mechanisms.

\subsubsection{Parity-even RNS amplitudes}

In the parity-even sector of the RNS computation, the functions $f^{(n)}$ arise from the summation over the even spin structures of the fermions on a genus-one worldsheet. We also take this opportunity to use the method of refs. [75, 76] to write down explicit results for the $N$-point spin sum for $N>7$.

Definition of $\boldsymbol{V}_{\boldsymbol{p}}\left(\boldsymbol{x}_{1}, \ldots, \boldsymbol{x}_{\boldsymbol{N}}\right)$. In the subsequent we use the variables $x_{i} \equiv z_{i}-z_{i+1}$ for $i=1, \ldots, N$ with the condition $z_{N+1}=z_{1}$ such that $\sum_{i=1}^{N} x_{i}=0$. Using the shorthand $\Omega_{i} \equiv \alpha \Omega\left(x_{i}, \alpha\right)$ it follows from eq. (3.30) that the $\alpha^{p}$-component of $\Omega_{1} \cdots \Omega_{N}$ has at most $p$ simultaneous single poles in the variables $x_{i}$. This suggests the following definition

$$
\left.V_{p}\left(x_{1}, x_{2}, \ldots, x_{N}\right) \equiv\left(\Omega_{1} \Omega_{2} \ldots \Omega_{N}\right)\right|_{\alpha^{p}} .
$$

For example, with $f_{i}^{(n)} \equiv f^{(n)}\left(x_{i}\right)$,

$$
V_{1}\left(x_{1}, \ldots, x_{5}\right)=\sum_{i=1}^{5} f_{i}^{(1)}
$$




$$
\begin{aligned}
V_{2}\left(x_{1}, \ldots, x_{6}\right) & =\sum_{i=1}^{6} f_{i}^{(2)}+\sum_{1 \leq i<j}^{6} f_{i}^{(1)} f_{j}^{(1)} \\
V_{3}\left(x_{1}, \ldots, x_{7}\right) & =\sum_{i=1}^{7} f_{i}^{(3)}+\sum_{1 \leq i<j}^{7}\left(f_{i}^{(1)} f_{j}^{(2)}+f_{i}^{(2)} f_{j}^{(1)}\right)+\sum_{1 \leq i<j<k}^{7} f_{i}^{(1)} f_{j}^{(1)} f_{k}^{(1)} \\
V_{4}\left(x_{1}, \ldots, x_{8}\right) & =\sum_{i=1}^{8} f_{i}^{(4)}+\sum_{1 \leq i<j}^{8}\left(f_{i}^{(1)} f_{j}^{(3)}+f_{i}^{(2)} f_{j}^{(2)}+f_{i}^{(3)} f_{j}^{(1)}\right)+\sum_{1 \leq i<j<k<l}^{8} f_{i}^{(1)} f_{j}^{(1)} f_{k}^{(1)} f_{l}^{(1)} \\
& +\sum_{1 \leq i<j<k}^{8}\left(f_{i}^{(1)} f_{j}^{(1)} f_{k}^{(2)}+f_{i}^{(1)} f_{j}^{(2)} f_{k}^{(1)}+f_{i}^{(2)} f_{j}^{(1)} f_{k}^{(1)}\right)
\end{aligned}
$$

Interestingly, the anti-holomorphic recursion eq. (3.27) implies that $V_{p}\left(x_{1}, \ldots, x_{N}\right)$ is holomorphic; $\frac{\partial}{\partial \bar{z}_{i}} V_{p}\left(x_{1}, \ldots, x_{N}\right)=0$. Equivalently, the non-holomorphic factors $\operatorname{Im}\left(x_{i}\right)$ in $V_{p}\left(x_{1}, \ldots, x_{N}\right)$ trivially vanish because of the condition $\sum_{i=1}^{N} x_{i}=0$. One can therefore replace $\mathcal{E}_{1}(x, \tau)$ by $E_{1}(x, \tau)$ and $f_{i}^{(n)} \rightarrow g_{i}^{(n)}$ in the notation of subsection 3.3.3 to establish manifest holomorphicity.

Note that the functions in eq. (5.8) were also used in [77] to cast one-loop correlation functions among arbitrary numbers of Kac-Moody currents into a closed form.

Spin sums in one-loop amplitudes. In the computation of parity-even one-loop amplitudes in the RNS formalism the bosonic worldsheet fields can be straightforwardly integrated out to yield products of $f^{(1)}$, possibly after integration by parts. Worldsheet fermions, on the other hand, give rise to the following spin sums,

$$
\mathcal{G}_{N}\left(x_{1}, \ldots, x_{N}\right) \equiv \sum_{\nu=1,2,3}(-1)^{\nu}\left(\frac{\theta_{\nu+1}(0, \tau)}{\theta_{1}^{\prime}(0, \tau)}\right)^{4} S_{\nu}\left(x_{1}\right) S_{\nu}\left(x_{2}\right) \ldots S_{\nu}\left(x_{N}\right)
$$

where $\sum_{i=1}^{N} x_{i}=0, S_{\nu}$ is the Szegö kernel and $\nu$ denotes the even spin structure with associated Jacobi theta functions $\theta_{2}, \theta_{3}, \theta_{4}[53,78-80]$,

$$
S_{\nu}(z) \equiv \frac{\theta_{1}^{\prime}(0, \tau) \theta_{\nu+1}(z, \tau)}{\theta_{\nu+1}(0, \tau) \theta_{1}(z, \tau)}
$$

A method to evaluate such sums was presented in ref. [75] and its explicit results at $N \leq 7$ can be written in terms of $f^{(1)}(z)$, the Weierstrass function $\wp(z)$ and its derivatives $\partial^{k} \wp(z)$,

$$
\begin{aligned}
& \mathcal{G}_{4}\left(x_{1}, \ldots, x_{4}\right)=1 \\
& \mathcal{G}_{5}\left(x_{1}, \ldots, x_{5}\right)=\sum_{j=1}^{5} f^{(1)}\left(x_{j}\right) \\
& \mathcal{G}_{6}\left(x_{1}, \ldots, x_{6}\right)=\frac{1}{2}\left\{\left(\sum_{j=1}^{6} f^{(1)}\left(x_{j}\right)\right)^{2}-\sum_{j=1}^{6} \wp\left(x_{j}\right)\right\} \\
& \mathcal{G}_{7}\left(x_{1}, \ldots, x_{7}\right)=\frac{1}{6}\left\{\left(\sum_{j=1}^{7} f^{(1)}\left(x_{j}\right)\right)^{3}-\sum_{j=1}^{7} \partial \wp\left(x_{j}\right)-3\left(\sum_{j=1}^{7} f^{(1)}\left(x_{j}\right)\right)\left(\sum_{j=1}^{7} \wp\left(x_{j}\right)\right)\right\} .
\end{aligned}
$$


One can show that the above results are naturally described by the elliptic functions $V_{p}\left(x_{1}, \ldots, x_{N}\right)$,

$$
\mathcal{G}_{N}\left(x_{1}, \ldots, x_{N}\right)=V_{N-4}\left(x_{1}, \ldots, x_{N}\right), \quad 4 \leq N \leq 7
$$

An alternative method was used in $[81,82]$ to express $\mathcal{G}_{N}$ in terms of single derivatives of the bosonic Green function. The equivalence of the expression for $\mathcal{G}_{6}$ given in these references with eq. (5.12) can be verified through the Fay identity eq. (2.39).

Although the results for $N \geq 8$ were not written down explicitly in ref. [75], they also take a natural form when expressed in terms of elliptic functions $V_{p}\left(x_{1}, \ldots, x_{N}\right)$,

$$
\begin{aligned}
\mathcal{G}_{8}\left(x_{1}, \ldots, x_{8}\right) & =V_{4}\left(x_{1}, \ldots, x_{8}\right)+3 e_{4} \\
\mathcal{G}_{9}\left(x_{1}, \ldots, x_{9}\right) & =V_{5}\left(x_{1}, \ldots, x_{9}\right)+3 e_{4} V_{1}\left(x_{1}, \ldots, x_{9}\right) \\
\mathcal{G}_{10}\left(x_{1}, \ldots, x_{10}\right) & =V_{6}\left(x_{1}, \ldots, x_{10}\right)+3 e_{4} V_{2}\left(x_{1}, \ldots, x_{10}\right)+10 e_{6} \\
\mathcal{G}_{11}\left(x_{1}, \ldots, x_{11}\right) & =V_{7}\left(x_{1}, \ldots, x_{11}\right)+3 e_{4} V_{3}\left(x_{1}, \ldots, x_{11}\right)+10 e_{6} V_{1}\left(x_{1}, \ldots, x_{11}\right) \\
\mathcal{G}_{12}\left(x_{1}, \ldots, x_{12}\right) & =V_{8}\left(x_{1}, \ldots, x_{12}\right)+3 e_{4} V_{4}\left(x_{1}, \ldots, x_{12}\right)+10 e_{6} V_{2}\left(x_{1}, \ldots, x_{12}\right)+42 e_{8} .
\end{aligned}
$$

The factors of the Eisenstein series $e_{j}$ eq. (3.9) can be systematically computed as well. Following ref. [76], we define $Q_{0}(\wp)=1, Q_{1}(\wp)=\wp$ and $Q_{k+1}(\wp)=\wp^{(2 k)}$. For example,

$$
\begin{aligned}
& Q_{2}(\wp)=3 ! \wp^{2}-\frac{1}{2} g_{2} \\
& Q_{3}(\wp)=5 ! \wp^{3}-18 g_{2} \wp-12 g_{3} \\
& Q_{4}(\wp)=7 ! \wp^{4}-1008 g_{2} \wp^{2}-720 g_{3} \wp+9 g_{2}^{2} \\
& Q_{5}(\wp)=9 ! \wp^{5}-90720 g_{2} \wp^{3}-64800 g_{3} \wp^{2}+3024 g_{2}^{2} \wp+2376 g_{2} g_{3},
\end{aligned}
$$

where the Weierstrass equation $\left(\wp^{\prime}\right)^{2}=4 \wp^{3}-g_{2} \wp-g_{3}$ has been used to rewrite the $2 k^{\text {th }}$ derivative of $\wp$ as a polynomial in $\wp$. In the above equation, $g_{2}=-4\left(s_{1} s_{2}+s_{2} s_{3}+s_{3} s_{1}\right)=$ $60 e_{4}, g_{3}=4 s_{1} s_{2} s_{3}=140 e_{6}$ are the elliptic invariants and $s_{i}$ are the branch points of the genus-one elliptic curve $y^{2}=4\left(z-s_{1}\right)\left(z-s_{2}\right)\left(z-s_{3}\right)$ satisfying $s_{1}+s_{2}+s_{3}=0$. Defining

$$
F_{2 k-4} \equiv-\frac{1}{(2 k-1) !} \frac{\left[\left(s_{1}-s_{3}\right) Q_{k}\left(s_{2}\right)+\left(s_{3}-s_{2}\right) Q_{k}\left(s_{1}\right)+\left(s_{2}-s_{1}\right) Q_{k}\left(s_{3}\right)\right]}{\left(s_{1}-s_{3}\right)\left(s_{3}-s_{2}\right)\left(s_{2}-s_{1}\right)}, \quad k \geq 4
$$

straightforward calculation leads to ${ }^{11}$

$$
F_{4}=3 e_{4}, \quad F_{6}=10 e_{6}, \quad F_{8}=42 e_{8}, \quad F_{10}=168 e_{10}, \quad F_{12}=627 e_{12}+9 e_{4}^{3},
$$

\footnotetext{
${ }^{11}$ The Eisenstein series $e_{8}, e_{10}$ and $e_{12}$ can be written in terms of $e_{4}$ and $e_{6}$ as follows

$$
e_{8}=\frac{3}{7} e_{4}^{2}, \quad e_{10}=\frac{5}{11} e_{4} e_{6}, \quad e_{12}=\frac{18}{143} e_{4}^{3}+\frac{25}{143} e_{6}^{2}
$$
}

The general formula is written in terms of $d_{k} \equiv(2 k+3) k ! e_{2 k+4}$

$$
d_{n+2}=\frac{3 n+6}{2 n+9} \sum_{k=0}^{n}\left(\begin{array}{l}
n \\
k
\end{array}\right) d_{k} d_{n-k} .
$$


which precisely captures the factors of $e_{j}$ in eq. (5.14). We have explicitly checked up to $N=12$ that the spin sums can be uniformly written as,

$$
\mathcal{G}_{N}\left(x_{1}, \ldots, x_{N}\right)=V_{N-4}\left(x_{1}, \ldots, x_{N}\right)+\sum_{k=1}^{\left\lfloor\frac{N-8}{2}\right\rfloor+1} F_{2 k+2} V_{N-2 k-6}\left(x_{1}, \ldots, x_{N}\right) .
$$

\subsubsection{Parity-odd RNS amplitudes}

The parity-odd sector of the RNS computation entirely stems from the unique odd spin structure at genus one where the worldsheet spinors obey anti-periodic boundary conditions along both torus cycles and acquire a zero mode. The worldsheet integrand is governed by zero-mode saturation and, probably as a common feature with the Green-Schwarz or pure spinor formalism, OPE contractions of the worldsheet fields which generate $N-4$ factors of $f_{i j}^{(1)}$ where $f_{i j}^{(n)} \equiv f^{(n)}\left(z_{i}-z_{j}\right)$.

For six points, the direct evaluation of the OPEs gives rise to a quadratic factor $f_{i j}^{(1)} f_{k l}^{(1)}$ for various combinations of labels capturing the behavior of the singularities as the vertices collide. However, we know from the Fay identity eq. (2.39) that these quadratic combinations are not linearly independent and therefore one is naturally led to higherweight $f^{(n)}$ 's when considering a minimal basis of integrals to evaluate. The simplest example where a higher-weight $f^{(n)}$ is generated this way is $f_{12}^{(1)} f_{13}^{(1)}+f_{23}^{(1)} f_{21}^{(1)}+f_{31}^{(1)} f_{32}^{(1)}=$ $f_{12}^{(2)}+f_{23}^{(2)}+f_{31}^{(2)}$ which can be viewed as generalizing the genus-zero partial fraction identity eq. (2.38). The non-vanishing of the right-hand side provides an important distinction between one-loop and tree-level string amplitudes and it is ultimately related to the gauge anomaly cancellation mechanism in the superstring $[57,58]$. It can be shown that the parity-odd part of the six-point amplitude as firstly computed in ref. [83] can be entirely written in terms of $f^{(2)}$, i.e. that any appearance of $f^{(1)}$ can be removed via eq. (2.39).

More generally, the $N-4$ powers of $f^{(1)}$ in the $N$-point amplitude allow, via the Fay identity, the generation of $f^{(p)}$ with up to $p=N-4$. In this way the need for a general integration method for the type of iterated integrals on an elliptic curve considered in this paper is justified.

\section{Discussion and further directions}

In this article, we have proposed an organization scheme for elliptic iterated integrals and elliptic multiple zeta values (eMZVs), where the key definitions are provided in eqs. (2.18) and (2.22). The infinite family of doubly-periodic functions $f^{(n)}$ appearing in the integrands of section 2 are put into a mathematical context and are related to multiple elliptic polylogarithms in section 3. As a first natural and simple application of this framework, we have identified eMZVs in the $\alpha^{\prime}$-expansion of one-loop scattering amplitudes in open string theory. The leading orders in the low-energy behavior of the four- and five-point amplitudes in terms of eMZVs are presented in eqs. (4.27) and (5.4). Divergent eMZVs turn out to cancel from our results.

Having demonstrated the potential of the formalism for an initial example, there are numerous open questions to be pursued in the near future. Most obviously, the eMZV 
content of the low energy expansion of cylinder amplitudes needs to be understood for higher orders in $\alpha^{\prime}$, which can be done conveniently using the new techniques. Furthermore, the contributions from the cylinder configuration with open string insertions on both boundaries as well as from the Möbius-strip topology shall be determined in terms of the iterated integrals introduced in subsection 2.2. The $q$-expansion of eMZVs exemplified in section 4.4.1 offers a promising approach to systematically perform the $t$-integration in eq. (4.1) after summing all topologies for the gauge group $\mathrm{SO}(32)$ [56].

On the mathematical side, the network of relations between eMZVs explored in subsection 2.2.3 will be further investigated in refs. [49, 67, 68]. A suitable coaction along the lines of refs. $[5-8,16]$ might lead to a natural basis choice for eMZVs and might allow to further identify patterns in the one-loop string amplitudes. In the same way as the Drinfeld associator was instrumental in understanding the pattern of MZVs [29] in open string tree-level amplitudes [37] and finally allowed to completely determine their $\alpha^{\prime}$-expansion in ref. [38], the elliptic associators discussed in ref. [24] might encode the structure of the $\alpha^{\prime}$-expansion at one-loop. Furthermore, in refs. [84, 85] so-called multiple modular values are discussed whose possible relation to the eMZVs studied here needs to be explored.

In multi-particle one-loop open string amplitudes, the pure spinor formalism, in particular the ingredients of ref. [86] are expected to yield a compact description of the kinematic factors associated to the functions $f^{(n)}$. While the precise superspace kinematic factors along with various powers of $f^{(1)}$ have been derived in ref. [69], the kinematic companions of $f^{(n \geq 2)}$ in the higher-point amplitudes are currently under investigation.

Finally, it would be desirable to find a similar scheme for organizing the $\alpha^{\prime}$-expansion of closed string one-loop amplitudes. In particular, the worldsheet integrals investigated in refs. [64-66] might allow for a description in terms of eMZVs and their counterpart defined with respect to the other cycle of the torus. The peculiar linear combinations of torus integrals appearing in the $\alpha^{\prime}$-expansion of closed-string amplitudes call for an explanation along the lines of the above finding that divergent eMZVs drop out from the open-string expansions.

\section{Acknowledgments}

OS and NM are grateful to the organizers of the conference "Numbers and Physics" in Madrid in September 2014, in particular to José Burgos for encouraging discussions. We would like to thank Francis Brown, Claude Duhr and Michael Green for comments and suggestions on the draft of this article as well as an unknown referee for his elaborate feedback on the first submitted version. JB wants to thank the Stanford Institute for Theoretical Physics, where part of this work was completed, for hospitality. Furthermore we are grateful to Francis Brown and Michael Green for helpful discussions and Ulf Kühn for valuable advice. JB, NM and CRM want to thank the Albert-Einstein-Institute for hospitality. OS is grateful to the Department of Applied Mathematics and Theoretical Physics of the University of Cambridge and to ETH Zürich for hospitality. CRM and OS acknowledge financial support by the European Research Council Advanced Grant No. 247252 of Michael Green. 


\section{A Derivatives of multiple polylogarithms w.r.t. the labels}

The proof of the recursion in eq. (2.11) relies on the derivatives of multiple polylogarithms eq. (2.1) with respect to their labels $a_{1}, a_{2}, \ldots, a_{n}[5]$ :

$$
\begin{aligned}
\frac{\partial}{\partial z} G(\vec{a} ; z) & =\frac{1}{z-a_{1}} G\left(a_{2}, \ldots, a_{n} ; z\right) . \\
\frac{\partial}{\partial a_{i}} G(\vec{a} ; z) & =\frac{1}{a_{i-1}-a_{i}} G\left(\ldots, \hat{a}_{i-1}, \ldots ; z\right)+\frac{1}{a_{i}-a_{i+1}} G\left(\ldots, \hat{a}_{i+1}, \ldots ; z\right) \\
& \quad-\frac{a_{i-1}-a_{i+1}}{\left(a_{i-1}-a_{i}\right)\left(a_{i}-a_{i+1}\right)} G\left(\ldots, \hat{a}_{i}, \ldots ; z\right), \quad i \neq 1, n \\
\frac{\partial}{\partial a_{n}} G(\vec{a} ; z) & =\frac{1}{a_{n-1}-a_{n}} G\left(\ldots, \hat{a}_{n-1}, a_{n} ; z\right)-\frac{a_{n-1}}{\left(a_{n-1}-a_{n}\right) a_{n}} G\left(\ldots, a_{n-1} ; z\right) .
\end{aligned}
$$

\section{B Identities for iterated integrals}

This appendix provides further relations to integrate eIIs whose argument occurs in the labels.

\section{B.1 Total derivatives}

The following identities generalize eqs. (2.34) to (2.36) for multiple successive occurrences of the argument $t_{0}$ in the label. If the first $k$ labels match the argument, one can show that

$$
\begin{aligned}
& \frac{\mathrm{d}}{\mathrm{d} t_{0}} \Gamma\left(\begin{array}{ccccccc}
n_{1} & n_{2} & \ldots & n_{k} & n_{k+1} & \ldots & n_{r} \\
t_{0} & t_{0} & \ldots & t_{0} & a_{k+1} & \ldots & a_{r}
\end{array} ; t_{0}\right) \\
& =\left(\prod_{j=1}^{k-1} \int_{0}^{t_{j-1}} \mathrm{~d} t_{j} f^{\left(n_{j}\right)}\left(t_{j}-t_{0}\right)\right) \int_{0}^{t_{k-1}} \mathrm{~d} t f^{\left(n_{k}\right)}\left(t-t_{0}\right) f^{\left(n_{k+1}\right)}\left(t-a_{k+1}\right) \Gamma\left(\begin{array}{ccc}
n_{k+2} & \ldots & n_{r} \\
a_{k+2} & \ldots & a_{r}
\end{array} ; t\right) .
\end{aligned}
$$

For a terminal sequence of $a_{j}=t_{0}$, we find

$$
\begin{aligned}
& \frac{\mathrm{d}}{\mathrm{d} t_{0}} \Gamma\left(\begin{array}{cccccc}
n_{1} & \ldots & n_{\ell-1} & n_{\ell} & \ldots & n_{r} \\
a_{1} & \ldots & a_{\ell-1} & t_{0} & \ldots & t_{0}
\end{array} ; t_{0}\right)=f^{\left(n_{1}\right)}\left(t_{0}-a_{1}\right) \Gamma\left(\begin{array}{cccccc}
n_{2} & \ldots & n_{\ell-1} & n_{\ell} & \ldots & n_{r} \\
a_{2} & \ldots & a_{\ell-1} & t_{0} & \ldots & t_{0}
\end{array} ; t_{0}\right) \\
& -\left(\prod_{j=1}^{\ell-2} \int_{0}^{t_{j-1}} \mathrm{~d} t_{j} f^{\left(n_{j}\right)}\left(t_{j}-a_{j}\right)\right) \int_{0}^{t_{\ell-2}} \mathrm{~d} t f^{\left(n_{\ell-1}\right)}\left(t-a_{\ell-1}\right) f^{\left(n_{\ell}\right)}\left(t-t_{0}\right) \Gamma\left(\begin{array}{ccc}
n_{\ell+1} & \ldots & n_{r} \\
t_{0} & \ldots & t_{0}
\end{array} ; t\right) \\
& +f^{\left(n_{r}\right)}\left(-t_{0}\right) \Gamma\left(\begin{array}{ccccccc}
n_{1} & \ldots & n_{\ell-1} & n_{\ell} & \ldots & n_{r-1} \\
a_{1} & \ldots & a_{\ell-1} & t_{0} & \ldots & t_{0}
\end{array} t_{0}\right) .
\end{aligned}
$$

Finally, an intermediate sequence of $a_{j}=t_{0}$ ranging from $j=p$ to $j=q$ with $p \neq 1$ and $q \neq r$ can be addressed via

$$
\begin{aligned}
& \frac{\mathrm{d}}{\mathrm{d} t_{0}} \Gamma\left(\begin{array}{ccccccccc}
n_{1} & \ldots & n_{p-1} & n_{p} & \ldots & n_{q} & n_{q+1} & \ldots & n_{r} \\
a_{1} & \ldots & a_{p-1} & t_{0} & \ldots & t_{0} & a_{q+1} & \ldots & a_{r}
\end{array} ; t_{0}\right)=f^{\left(n_{1}\right)}\left(t_{0}-a_{1}\right) \Gamma\left(\begin{array}{ccccccccc}
n_{2} & \ldots & n_{p-1} & n_{p} & \ldots & n_{q} & n_{q+1} & \ldots & n_{r} \\
a_{2} & \ldots & a_{p-1} & t_{0} & \ldots & t_{0} & a_{q+1} & \ldots & a_{r}
\end{array} ; t_{0}\right) \\
& -\left(\prod_{j=1}^{p-2} \int_{0}^{t_{j-1}} \mathrm{~d} t_{j} f^{\left(n_{j}\right)}\left(t_{j}-a_{j}\right)\right)
\end{aligned}
$$




$$
\begin{aligned}
& \times \int_{0}^{t_{p-2}} \mathrm{~d} t f^{\left(n_{p-1}\right)}\left(t-a_{p-1}\right) f^{\left(n_{p}\right)}\left(t-t_{0}\right) \Gamma\left(\begin{array}{cccccc}
n_{p+1} & \ldots & n_{q} & n_{q+1} & \ldots & n_{r} \\
t_{0} & \ldots & t_{0} & a_{q+1} & \ldots & a_{r}
\end{array} ; t\right) \\
& +\left(\prod_{j=1}^{p-1} \int_{0}^{t_{j-1}} \mathrm{~d} t_{j} f^{\left(n_{j}\right)}\left(t_{j}-a_{j}\right)\right)\left(\prod_{j=p}^{q-1} \int_{0}^{t_{j-1}} \mathrm{~d} t_{j} f^{\left(n_{j}\right)}\left(t_{j}-t_{0}\right)\right) \\
& \times \int_{0}^{t_{q-1}} \mathrm{~d} t f^{\left(n_{q}\right)}\left(t-t_{0}\right) f^{\left(n_{q+1}\right)}\left(t-a_{q+1}\right) \Gamma\left(\begin{array}{ccc}
n_{q+2} & \ldots & n_{r} \\
a_{q+2} & \ldots & a_{r}
\end{array} t\right) .
\end{aligned}
$$

Cases with multiple disconnected sequences of $a_{j}=t_{0}$ can be treated along similar lines.

\section{B.2 Recursive removal of the argument from the labels}

On the basis of eqs. (B.1) to (B.3), we can generalize the recursions eqs. (2.41) to (2.43) to situations where several successive instances of the argument occur among the labels. If the first $k$ labels match the argument, one can show that

$$
\begin{aligned}
& \Gamma\left(\begin{array}{ccccccc}
n_{1} & n_{2} & \ldots & n_{k} & n_{k+1} & \ldots & n_{r} \\
z & z & \ldots & z & a_{k+1} & \ldots & a_{r}
\end{array} ; z\right)=\lim _{z \rightarrow 0} G\left(z, \ldots, z, a_{k+1}, \ldots, a_{r} ; z\right) \prod_{j=1}^{r} \delta_{n_{j}, 1} \\
& -(-1)^{n_{k}} \int_{0}^{z} \mathrm{~d} t f^{\left(n_{k}+n_{k+1}\right)}\left(t-a_{k+1}\right) \Gamma\left(\begin{array}{cccccccc}
n_{1} & \ldots & n_{k-1} & 0 & n_{k+2} & \ldots & n_{r} \\
t & \ldots & t & 0 & a_{k+2} & \ldots & a_{r}
\end{array} ; t\right)
\end{aligned}
$$

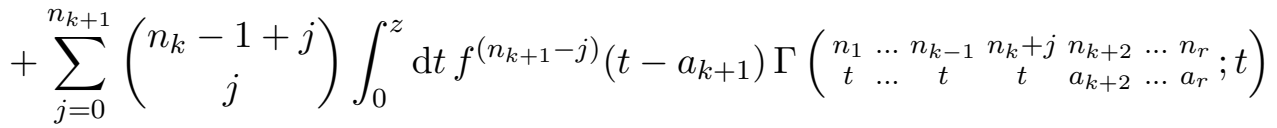

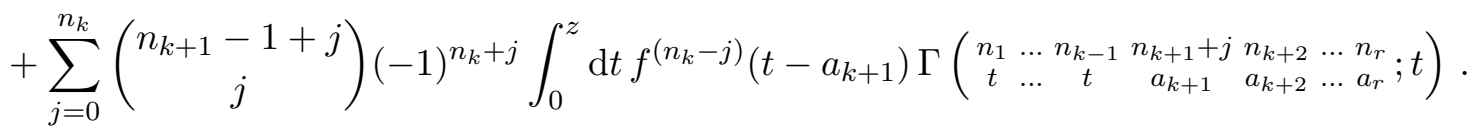

For a terminal sequence of $a_{j}=z$, we find

$$
\begin{aligned}
& \Gamma\left(\begin{array}{ccccccc}
n_{1} & \ldots & n_{\ell-1} & n_{\ell} & \ldots & n_{r} \\
a_{1} & \ldots & a_{\ell-1} & z & \ldots & z
\end{array} ; z\right)=\lim _{z \rightarrow 0} G\left(a_{1}, \ldots, a_{\ell-1}, z, \ldots, z ; z\right) \prod_{j=1}^{r} \delta_{n_{j}, 1} \\
& +\int_{0}^{z} \mathrm{~d} t f^{\left(n_{1}\right)}\left(t-a_{1}\right) \Gamma\left(\begin{array}{cccccc}
n_{2} & \ldots & n_{\ell-1} & n_{\ell} & \ldots & n_{r} \\
a_{2} & \ldots & a_{\ell-1} & t & \ldots & t
\end{array} ; t\right) \\
& +(-1)^{n_{\ell}} \int_{0}^{z} \mathrm{~d} t f^{\left(n_{\ell}+n_{\ell-1}\right)}\left(t-a_{\ell-1}\right) \Gamma\left(\begin{array}{cccccccc}
n_{1} & \ldots & n_{\ell-2} & 0 & n_{\ell+1} & \ldots & n_{r} \\
a_{1} & \ldots & a_{\ell-2} & 0 & t & \ldots & \ldots & t
\end{array} ; t\right)
\end{aligned}
$$

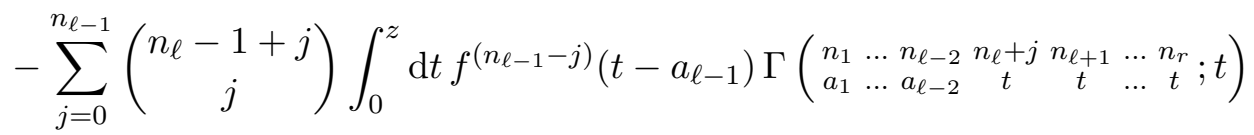

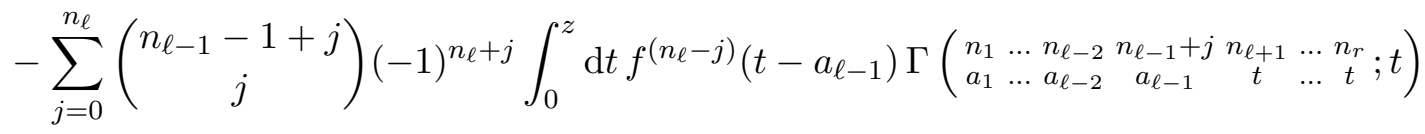

$$
\begin{aligned}
& +(-1)^{n_{r}} \int_{0}^{z} \mathrm{~d} t f^{\left(n_{r}\right)}(t) \Gamma\left(\begin{array}{cccccc}
n_{1} & \ldots & n_{\ell-1} & n_{\ell} & \ldots & n_{r-1} \\
a_{1} & \ldots & a_{\ell-1} & t & \ldots & t
\end{array} ; t\right) .
\end{aligned}
$$

Finally, an intermediate sequence of $a_{j}=z$ ranging from $j=p$ to $j=q$ with $p \neq 1$ and $q \neq r$ can be addressed via

$$
\Gamma\left(\begin{array}{ccccccccc}
n_{1} & \ldots & n_{p-1} & n_{p} & \ldots & n_{q} & n_{q+1} & \ldots & n_{r} \\
a_{1} & \ldots & a_{p-1} & z & \ldots & z & a_{q+1} & \ldots & a_{r}
\end{array} ; z\right)=\lim _{z \rightarrow 0} G\left(a_{1}, \ldots, a_{p-1}, z, \ldots, z, a_{q+1}, \ldots, a_{r} ; z\right) \prod_{j=1}^{r} \delta_{n_{j}, 1}
$$




$$
\begin{aligned}
& +\int_{0}^{z} \mathrm{~d} t f^{\left(n_{1}\right)}\left(t-a_{1}\right) \Gamma\left(\begin{array}{cccccccccc}
n_{2} & \ldots & n_{p-1} & n_{p} & \ldots & n_{q} & n_{q+1} & \ldots & n_{r} \\
a_{2} & \ldots & a_{p-1} & t & \ldots & t & a_{q+1} & \ldots & a_{r}
\end{array} ; t\right) \\
& +(-1)^{n_{p}} \int_{0}^{z} \mathrm{~d} t f^{\left(n_{p}+n_{p-1}\right)}\left(t-a_{p-1}\right) \Gamma\left(\begin{array}{cccccccccc}
n_{1} & \ldots & n_{p-2} & 0 & n_{p+1} & \ldots & n_{q} & n_{q+1} & \ldots & n_{r} \\
a_{1} & \ldots & a_{p-2} & 0 & t & \ldots & t & a_{q+1} & \ldots & a_{r}
\end{array} ;\right)
\end{aligned}
$$

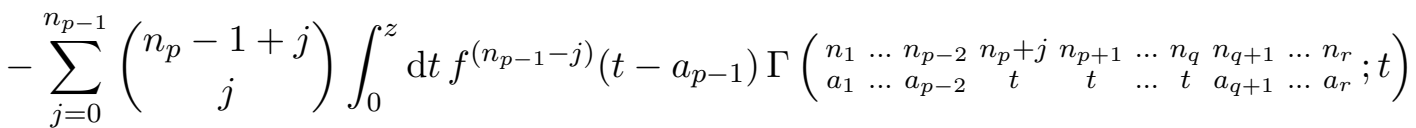

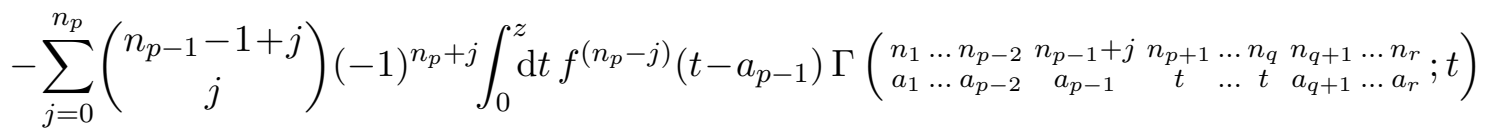

$$
\begin{aligned}
& -(-1)^{n_{q}} \int_{0}^{z} \mathrm{~d} t f^{\left(n_{q}+n_{q+1}\right)}\left(t-a_{q+1}\right) \Gamma\left(\begin{array}{cccccccccc}
n_{1} & \ldots & n_{p-1} & n_{p} & \ldots & n_{q-1} & 0 & n_{q+2} & \ldots & n_{r} \\
a_{1} & \ldots & a_{p-1} & t & \ldots & t & 0 & a_{q+2} & \ldots & a_{r}
\end{array} ; t\right)
\end{aligned}
$$

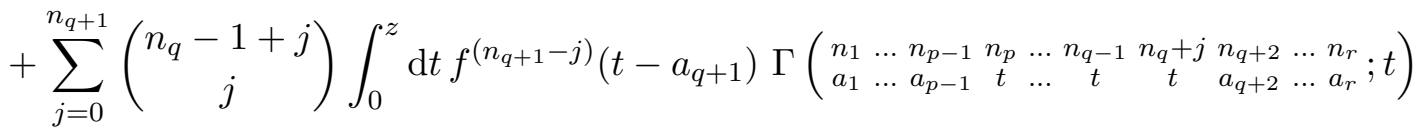

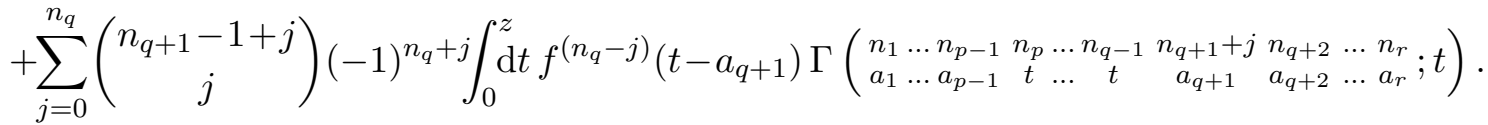

These relations reproduce eqs. (2.41) to (2.43) for $k=1, p=q$ and $\ell=r$, respectively.

\section{B.3 Eliminating labels $a_{j}=z$ at length three}

The generalization of eq. (2.52) to length three is governed by

$$
\begin{aligned}
& \Gamma\left(\begin{array}{ccc}
n_{1} & n_{2} & n_{3} \\
z & 0 & 0
\end{array} ; z\right)=-\zeta_{3} \delta_{n_{1}}^{1} \delta_{n_{2}}^{1} \delta_{n_{3}}^{1}+\zeta_{2} \sum_{j=0}^{n_{2}} \delta_{n_{3}}^{1} \delta_{n_{1}+j}^{1}\left(\begin{array}{c}
n_{1}-1+j \\
j
\end{array}\right) \Gamma\left(n_{2}-j ; z\right) \\
& -(-1)^{n_{1}} \Gamma\left(n_{1}+n_{2}, 0, n_{3} ; z\right)+\sum_{j=0}^{n_{1}}(-1)^{n_{1}+j}\left(\begin{array}{c}
n_{2}-1+j \\
j
\end{array}\right) \Gamma\left(n_{1}-j, n_{2}+j, n_{3} ; z\right) \\
& -\sum_{j=0}^{n_{2}}(-1)^{n_{1}+j}\left(\begin{array}{c}
n_{1}-1+j \\
j
\end{array}\right) \Gamma\left(n_{2}-j, n_{1}+n_{3}+j, 0 ; z\right) \\
& +\sum_{j=0}^{n_{2}}\left(\begin{array}{c}
n_{1}-1+j \\
j
\end{array}\right) \sum_{k=0}^{n_{3}}(-1)^{n_{1}+j+k}\left(\begin{array}{c}
n_{1}+j-1+k \\
k
\end{array}\right) \Gamma\left(n_{2}-j, n_{3}-k, n_{1}+j+k ; z\right) \\
& +\sum_{j=0}^{n_{2}}\left(\begin{array}{c}
n_{1}-1+j \\
j
\end{array}\right) \sum_{k=0}^{n_{1}+j}(-1)^{n_{1}+j+k}\left(\begin{array}{c}
n_{3}-1+k \\
k
\end{array}\right) \Gamma\left(n_{2}-j, n_{1}+j-k, n_{3}+k ; z\right) .
\end{aligned}
$$

The reflection identity (2.20) allows to infer $\Gamma\left(\begin{array}{ccc}n_{1} & n_{2} & n_{3} \\ z & z & 0\end{array} ; z\right)=(-1)^{n_{1}+n_{2}+n_{3}} \Gamma\left(\begin{array}{ccc}n_{3} & n_{2} & n_{1} \\ z & 0 & 0\end{array} ; z\right)$, and permutations in the labels are covered by shuffle relations.

\section{Trigonometric integrals}

This appendix gathers trigonometric integrals relevant for the evaluation of eMZVs. The result in eq. (4.30) for $\omega(0,1,0,0)$ relies on

$$
\int_{0}^{1} \mathrm{~d} z_{4} \int_{0}^{z_{4}} \mathrm{~d} z_{3} \int_{0}^{z_{3}} \mathrm{~d} z_{2} \sin \left(2 \pi n z_{2}\right) z_{2}=\frac{3}{8 \pi^{3} n^{3}}
$$




$$
\int_{0}^{1} \mathrm{~d} z_{4} \int_{0}^{z_{4}} \mathrm{~d} z_{3} \int_{0}^{z_{3}} \mathrm{~d} z_{2} \cot \left(\pi z_{2}\right) z_{2}=\frac{3 \zeta_{3}}{4 \pi^{3}}
$$

and the eMZVs relevant at order $s_{i j}^{2}$ as given by eq. (4.31) and eq. (4.32) are based on

$$
\begin{aligned}
\int_{0}^{1} \mathrm{~d} z_{5} \int_{0}^{z_{5}} \mathrm{~d} z_{4} \int_{0}^{z_{4}} \mathrm{~d} z_{3} \int_{0}^{z_{3}} \mathrm{~d} z_{2} \int_{0}^{z_{2}} \mathrm{~d} z_{1} \cos \left(2 \pi n z_{1}\right) & =\frac{1}{24 \pi^{2} n^{2}}-\frac{1}{16 \pi^{4} n^{4}} \\
\int_{0}^{1} \mathrm{~d} z_{5} \int_{0}^{z_{5}} \mathrm{~d} z_{4} \int_{0}^{z_{4}} \mathrm{~d} z_{3} \int_{0}^{z_{3}} \mathrm{~d} z_{2} \cos \left(2 \pi n z_{2}\right) z_{2} & =-\frac{1}{24 \pi^{2} n^{2}}+\frac{1}{4 \pi^{4} n^{4}}
\end{aligned}
$$

\section{Cycle index of the symmetric group and the $f^{(n)}$ functions}

This appendix highlights the connection between the explicit expansion of the doublyperiodic functions $f^{(n)}$ in $(3.23)$ with the cycle index of the symmetric group $S_{n}$. For general references, see $[87,88]$.

Cycle structures. Every permutation $g \in S_{n}$ of $X=\{1, \ldots, n\}$ can be written as the product of disjoint cycles with lengths $a_{1}, \ldots, a_{n}$ such that $n=\sum_{i=1}^{n} a_{i}$. This integer partition of $n$ is represented by $\lambda=1^{a_{1}} 2^{a_{2}} \ldots n^{a_{n}}$ and is called the cycle structure of the permutation. Therefore the total number of cycle structures for the permutations in $S_{n}$ is given by the integer partition $P(n)=1,2,3,5,7, \ldots$. Note that the number of terms in each $f^{(n)}$ is also $P(n)$. Furthermore, if $\lambda=1^{a_{1}} 2^{a_{2}} \ldots n^{a_{n}}$ is a partition of $n$ (denoted by $\lambda \vdash n$ ), the number of permutations with cycle structure $\lambda$ is [87]

$$
\frac{n !}{\prod_{i=1}^{n} i^{a_{i}} a_{i} !} .
$$

Note that the coefficients of the monomials $\mathcal{E}_{1}^{a_{1}} \ldots \mathcal{E}_{n}^{a_{n}}$ in $f^{(n)}$ given by eq. (3.24) are reproduced by the formula (D.1) with the corresponding cycle structure. This observation can be made more precise with the definition of the cycle index of the symmetric group $S_{n}$ [87],

$$
Z\left(S_{n} ; s_{1}, \ldots, s_{n}\right)=\frac{1}{n !} \sum_{g \in S_{n}} z\left(g ; s_{1}, \ldots, s_{n}\right),
$$

where $z\left(g ; s_{1}, \ldots, s_{n}\right)=s_{1}^{a_{1}} s_{2}^{a_{2}} \ldots s_{n}^{a_{n}}$ and $a_{i}$ counts the number of cycles of length $i$ in the permutation $g$. One can see from the first few examples, ${ }^{12}$

$$
\begin{aligned}
Z\left(S_{1}, s_{1}\right) & =s_{1} \\
Z\left(S_{2}, s_{1}, \ldots, s_{2}\right) & =\frac{1}{2 !}\left(s_{1}^{2}+s_{2}\right) \\
Z\left(S_{3}, s_{1}, \ldots, s_{3}\right) & =\frac{1}{3 !}\left(s_{1}^{3}+3 s_{1} s_{2}+2 s_{3}\right) \\
Z\left(S_{4}, s_{1}, \ldots, s_{4}\right) & =\frac{1}{4 !}\left(s_{1}^{4}+6 s_{1}^{2} s_{2}+8 s_{1} s_{3}+3 s_{2}^{2}+6 s_{4}\right)
\end{aligned}
$$

\footnotetext{
${ }^{12}$ In addition, it is convenient to define $Z\left(S_{0}\right)=1$.
} 
that the cycle index of $S_{n}$ captures the expansions in (3.23). More precisely, theorem 1.3.3 of [89] can be written as

$$
\sum_{n=0}^{\infty} \alpha^{n} Z\left(S_{n} ; \mathcal{E}_{1}, \ldots, \mathcal{E}_{n}\right)=\exp \left(\sum_{j=1}^{\infty} \frac{\mathcal{E}_{j}}{j} \alpha^{j}\right),
$$

and comparing (3.22) with (D.3) leads to,

$$
\begin{aligned}
f^{(n)} & =Z\left(S_{n} ; \mathcal{E}_{1}, \ldots, \mathcal{E}_{n}\right) \\
& =\sum_{\lambda \vdash n} \prod_{i=1}^{n} \frac{\mathcal{E}_{i}^{a_{i}}}{i^{a_{i}} a_{i} !}, \quad \lambda=1^{a_{1}} 2^{a_{2}} \ldots n^{a_{n}} .
\end{aligned}
$$

Furthermore, one can also show that [88],

$$
\frac{\partial f^{(n)}(z, \tau)}{\partial \mathcal{E}_{p}}=\frac{1}{p} f^{(n-p)}(z, \tau) .
$$

Note, in particular, that (D.5) yields an alternative proof of (3.27),

$$
\frac{\partial f^{(n)}(z, \tau)}{\partial \bar{z}}=\frac{\partial f^{(n)}(z, \tau)}{\partial \mathcal{E}_{1}} \frac{\partial \mathcal{E}_{1}}{\partial \bar{z}}=-\frac{\pi}{\operatorname{Im}(\tau)} f^{(n-1)}(z, \tau) .
$$

Symmetric polynomials. The cycle index of the symmetric group $S_{n}$ also provides a recipe for expressing the complete symmetric function $h_{j}$ in terms of the power sum function $p_{j}$, i.e., $h_{n}=Z\left(S_{n} ; p_{1}, p_{2}, \ldots, p_{n}\right)$ [87]. Therefore the functional form of $h_{n}$ matches that of $f^{(n)}$ and one can exploit the well-known relation $n h_{n}=\sum_{i=1}^{n} p_{i} h_{n-i}$ from the theory of symmetric functions to obtain the corresponding recursion formula eq. (3.25) for $f^{(n)}$.

Open Access. This article is distributed under the terms of the Creative Commons Attribution License (CC-BY 4.0), which permits any use, distribution and reproduction in any medium, provided the original author(s) and source are credited.

\section{References}

[1] A.B. Goncharov, M. Spradlin, C. Vergu and A. Volovich, Classical polylogarithms for amplitudes and Wilson loops, Phys. Rev. Lett. 105 (2010) 151605 [arXiv:1006.5703] [INSPIRE].

[2] L.J. Dixon, C. Duhr and J. Pennington, Single-valued harmonic polylogarithms and the multi-Regge limit, JHEP 10 (2012) 074 [arXiv:1207.0186] [INSPIRE].

[3] J. Broedel, O. Schlotterer and S. Stieberger, Polylogarithms, multiple zeta values and superstring amplitudes, Fortsch. Phys. 61 (2013) 812 [arXiv:1304.7267] [INSPIRE].

[4] V. Del Duca, L.J. Dixon, C. Duhr and J. Pennington, The BFKL equation, Mueller-Navelet jets and single-valued harmonic polylogarithms, JHEP 02 (2014) 086 [arXiv:1309.6647] [INSPIRE].

[5] A.B. Goncharov, Multiple polylogarithms and mixed Tate motives, math/0103059 [INSPIRE]. 
[6] A.B. Goncharov, Galois symmetries of fundamental groupoids and noncommutative geometry, Duke Math. J. 128 (2005) 209 [math/0208144] [INSPIRE].

[7] F. Brown, On the decomposition of motivic multiple zeta values, in Galois-Teichmüller theory and arithmetic geometry, Math. Soc. Japan, Tokyo Japan (2012), pg. 31 [arXiv:1102.1310] [INSPIRE].

[8] C. Duhr, Hopf algebras, coproducts and symbols: an application to Higgs boson amplitudes, JHEP 08 (2012) 043 [arXiv: 1203.0454] [INSPIRE].

[9] L. Adams, C. Bogner and S. Weinzierl, The two-loop sunrise graph with arbitrary masses, J. Math. Phys. 54 (2013) 052303 [arXiv:1302.7004] [INSPIRE].

[10] S. Bloch and P. Vanhove, The elliptic dilogarithm for the sunset graph, J. Number Theor. 148 (2015) 328 [arXiv:1309.5865] [INSPIRE].

[11] L. Adams, C. Bogner and S. Weinzierl, The two-loop sunrise graph in two space-time dimensions with arbitrary masses in terms of elliptic dilogarithms, J. Math. Phys. 55 (2014) 102301 [arXiv:1405.5640] [inSPIRE].

[12] F. Brown and O. Schnetz, A K3 in $\phi^{4}$, Duke Math. J. 161 (2012) 1817 [arXiv:1006.4064] [INSPIRE].

[13] F. Brown and D. Doryn, Framings for graph hypersurfaces, arXiv:1301.3056 [INSPIRE].

[14] S. Caron-Huot and K.J. Larsen, Uniqueness of two-loop master contours, JHEP 10 (2012) 026 [arXiv:1205.0801] [InSPIRE].

[15] S. Bloch, M. Kerr and P. Vanhove, A Feynman integral via higher normal functions, arXiv:1406.2664 [INSPIRE].

[16] F. Brown and A. Levin, Multiple elliptic polylogarithms, arXiv:1110.6917.

[17] B. Enriquez, Analogues elliptiques des nombres multizétas (in French), arXiv:1301.3042.

[18] A. Beilinson and A. Levin, The elliptic polylogarithm, in Proc. of Symp. in Pure Math. 55, Part II, J.-P.S.U. Jannsen and S.L. Kleiman eds., AMS, U.S.A. (1994), pg. 123.

[19] S.J. Bloch, Higher regulators, algebraic K-theory, and zeta functions of elliptic curves, American Mathematical Society, Providence RI U.S.A. (2000), pg. 1.

[20] A. Levin, Elliptic polylogarithms: an analytic theory, Compos. Math. 106 (1997) 267.

[21] J. Wildeshaus, Realizations of polylogarithms, Springer, Germany (1997).

[22] D. Zagier, The Bloch-Wigner-Ramakrishnan polylogarithm function, Math. Ann. 286 (1990) 613.

[23] A. Weil, Elliptic functions according to Eisenstein and Kronecker, published in Ergebnisse der Mathematik und ihrer Grenzgebiete, Springer, Heidelberg Germany (1976).

[24] B. Enriquez, Elliptic associators, Select. Math. (N.S.) 20 (2014) 491.

[25] V. Drinfeld, Quasi Hopf algebras, Leningrad Math. J. 1 (1989) 1419.

[26] V. Drinfeld, On quasitriangular quasi-Hopf algebras and on a group that is closely connected with $\operatorname{Gal}(\overline{\mathbb{Q}} / \mathbb{Q})$, Leningrad Math. J. 2 (1991) 829.

[27] T. Le and J. Murakami, Kontsevich's integral for the Kauffman polynomial, Nagoya Math. J. 142 (1996) 39. 
[28] S. Stieberger, Constraints on tree-level higher order gravitational couplings in superstring theory, Phys. Rev. Lett. 106 (2011) 111601 [arXiv:0910.0180] [INSPIRE].

[29] O. Schlotterer and S. Stieberger, Motivic multiple zeta values and superstring amplitudes, J. Phys. A 46 (2013) 475401 [arXiv:1205.1516] [InSPIRE].

[30] O. Schnetz, Graphical functions and single-valued multiple polylogarithms, Commun. Num. Theor. Phys. 08 (2014) 589 [arXiv:1302.6445] [INSPIRE].

[31] F. Brown, Single-valued motivic periods and multiple zeta values, SIGMA 2 (2014) e25 [arXiv: 1309.5309] [INSPIRE].

[32] S. Stieberger, Closed superstring amplitudes, single-valued multiple zeta values and the Deligne associator, J. Phys. A 47 (2014) 155401 [arXiv:1310.3259] [inSPIRE].

[33] S. Stieberger and T.R. Taylor, Closed string amplitudes as single-valued open string amplitudes, Nucl. Phys. B 881 (2014) 269 [arXiv:1401.1218] [InSPIRE].

[34] D. Oprisa and S. Stieberger, Six gluon open superstring disk amplitude, multiple hypergeometric series and Euler-Zagier sums, hep-th/0509042 [INSPIRE].

[35] S. Stieberger and T.R. Taylor, Multi-gluon scattering in open superstring theory, Phys. Rev. D 74 (2006) 126007 [hep-th/0609175] [INSPIRE].

[36] T. Terasoma, Selberg integrals and multiple zeta values, Compos. Math. 133 (2002) 1 [math/9908045].

[37] J.M. Drummond and É. Ragoucy, Superstring amplitudes and the associator, JHEP 08 (2013) 135 [arXiv:1301.0794] [INSPIRE].

[38] J. Broedel, O. Schlotterer, S. Stieberger and T. Terasoma, All order $\alpha^{\prime}$-expansion of superstring trees from the Drinfeld associator, Phys. Rev. D 89 (2014) 066014 [arXiv: 1304.7304] [INSPIRE].

[39] $\alpha^{\prime}$-expansion of open superstring amplitudes website, http://mzv.mpp.mpg.de.

[40] M.B. Green, J. Schwarz and E. Witten, Superstring theory. Vol. 2: loop amplitudes, anomalies and phenomenology, Cambridge Monographs on Mathematical Physics, Cambridge Univ. Pr., Cambridge U.K. (1987).

[41] C. Duhr, H. Gangl and J.R. Rhodes, From polygons and symbols to polylogarithmic functions, JHEP 10 (2012) 075 [arXiv: 1110.0458] [INSPIRE].

[42] J. Ablinger, J. Blümlein and C. Schneider, Analytic and algorithmic aspects of generalized harmonic sums and polylogarithms, J. Math. Phys. 54 (2013) 082301 [arXiv:1302.0378] [INSPIRE].

[43] J. Ablinger and J. Blümlein, Harmonic sums, polylogarithms, special numbers and their generalizations, arXiv:1304.7071 [INSPIRE].

[44] J.M. Borwein, D.M. Bradley, D.J. Broadhurst and P. Lisonek, Special values of multiple polylogarithms, Trans. Amer. Math. Soc. 353 (2001) 907 [math/9910045] [INSPIRE].

[45] F. Brown, Mixed Tate motives over $\mathbb{Z}$, Ann. Math. 175 (2012) 949.

[46] F.C.S. Brown, Multiple zeta values and periods of moduli spaces $\mathfrak{M}_{0, n}$, Annales Sci. Ecole Norm. Sup. 42 (2009) 371 [math/0606419] [INSPIRE].

[47] C. Bogner and F. Brown, Feynman integrals and iterated integrals on moduli spaces of curves of genus zero, Commun. Num. Theor. Phys. 09 (2015) 189 [arXiv:1408.1862] [INSPIRE]. 
[48] J. Blumlein, D.J. Broadhurst and J.A.M. Vermaseren, The multiple zeta value data mine, Comput. Phys. Commun. 181 (2010) 582 [arXiv:0907.2557] [InSPIRE].

[49] J. Broedel, N. Matthes and O. Schlotterer, Relations between elliptic multiple zeta values and a special derivation algebra, arXiv:1507.02254 [INSPIRE].

[50] A. Levin and G. Racinet, Towards multiple elliptic polylogarithms, math/0703237.

[51] L. Kronecker, Zur Theorie der elliptischen Funktionen (in German), Mathematische Werke IV (1881) 313.

[52] D. Zagier, Periods of modular forms and Jacobi theta functions, Invent. Math. 104 (1991) 449.

[53] D. Mumford, M. Nori and P. Norman, Tata lectures on theta I, Birkhäuser, U.S.A. (1983).

[54] D. Mumford, M. Nori and P. Norman, Tata lectures on theta II, Birkhäuser, U.S.A. (1984).

[55] R. Hain, Notes on the universal elliptic KZB equation, arXiv:1309.0580.

[56] M.B. Green and J.H. Schwarz, Infinity cancellations in $\mathrm{SO}(32)$ superstring theory, Phys. Lett. B 151 (1985) 21 [inSPIRE].

[57] M.B. Green and J.H. Schwarz, Anomaly cancellation in supersymmetric D $=10$ gauge theory and superstring theory, Phys. Lett. B 149 (1984) 117 [INSPIRE].

[58] M.B. Green and J.H. Schwarz, The hexagon gauge anomaly in type I superstring theory, Nucl. Phys. B 255 (1985) 93 [inSPIRE].

[59] M.B. Green and J.H. Schwarz, Supersymmetrical dual string theory. 3. Loops and renormalization, Nucl. Phys. B 198 (1982) 441 [INSPIRE].

[60] J.H. Schwarz, Superstring theory, Phys. Rept. 89 (1982) 223 [INSPIRE].

[61] M.B. Green, J. Schwarz and E. Witten, Superstring theory. Vol. 1: introduction, Cambridge Monographs on Mathematical Physics, Cambridge Univ. Pr., Cambridge U.K. (1987).

[62] M.B. Green, J.H. Schwarz and L. Brink, $N=4$ Yang-Mills and $N=8$ supergravity as limits of string theories, Nucl. Phys. B 198 (1982) 474 [INSPIRE].

[63] M.B. Green and P. Vanhove, The low-energy expansion of the one loop type-II superstring amplitude, Phys. Rev. D 61 (2000) 104011 [hep-th/9910056] [INSPIRE].

[64] M.B. Green, J.G. Russo and P. Vanhove, Low energy expansion of the four-particle genus-one amplitude in type-II superstring theory, JHEP 02 (2008) 020 [arXiv:0801.0322] [INSPIRE].

[65] D.M. Richards, The one-loop five-graviton amplitude and the effective action, JHEP 10 (2008) 042 [arXiv:0807.2421] [INSPIRE].

[66] M.B. Green, C.R. Mafra and O. Schlotterer, Multiparticle one-loop amplitudes and S-duality in closed superstring theory, JHEP 10 (2013) 188 [arXiv:1307.3534] [INSPIRE].

[67] N. Matthes, Elliptic double zeta values, in preparation.

[68] N. Matthes, work in progress.

[69] C.R. Mafra and O. Schlotterer, The structure of n-point one-loop open superstring amplitudes, JHEP 08 (2014) 099 [arXiv: 1203.6215] [INSPIRE].

[70] Z. Bern, J.J.M. Carrasco and H. Johansson, New relations for gauge-theory amplitudes, Phys. Rev. D 78 (2008) 085011 [arXiv:0805.3993] [INSPIRE]. 
[71] N. Berkovits, Super Poincaré covariant quantization of the superstring, JHEP 04 (2000) 018 [hep-th/0001035] [INSPIRE].

[72] P. Ramond, Dual theory for free fermions, Phys. Rev. D 3 (1971) 2415 [InSPIRE].

[73] A. Neveu and J.H. Schwarz, Factorizable dual model of pions, Nucl. Phys. B 31 (1971) 86 [INSPIRE].

[74] A. Neveu and J.H. Schwarz, Quark model of dual pions, Phys. Rev. D 4 (1971) 1109 [INSPIRE].

[75] A. Tsuchiya, More on one loop massless amplitudes of superstring theories, Phys. Rev. D 39 (1989) 1626 [inSPIRE].

[76] A.G. Tsuchiya, On the pole structures of the disconnected part of hyper elliptic g-loop $M$-point super string amplitudes, arXiv:1209.6117 [INSPIRE].

[77] L. Dolan and P. Goddard, Current algebra on the torus, Commun. Math. Phys. 285 (2009) 219 [arXiv:0710.3743] [INSPIRE].

[78] M.A. Namazie, K.S. Narain and M.H. Sarmadi, On loop amplitudes in the fermionic string, RAL-86-051, (1986) [INSPIRE].

[79] J. Igusa, Theta functions, Springer, Germany (1972).

[80] J. Fay, Theta functions on Riemann surfaces, Springer, Germany (1973).

[81] S. Stieberger and T.R. Taylor, Non-Abelian Born-Infeld action and type-I heterotic duality (I): heterotic $F^{6}$ terms at two loops, Nucl. Phys. B 647 (2002) 49 [hep-th/0207026] [INSPIRE].

[82] S. Stieberger and T.R. Taylor, Non-Abelian Born-Infeld action and type-I heterotic duality (II): nonrenormalization theorems, Nucl. Phys. B 648 (2003) 3 [hep-th/0209064] [INSPIRE].

[83] L. Clavelli, P.H. Cox and B. Harms, Parity violating one loop six point function in type-I superstring theory, Phys. Rev. D 35 (1987) 1908 [INSPIRE].

[84] F. Brown, Motivic periods and the projective line minus three points, in Proceedings of the ICM 2014, Seoul Korea (2014) [arXiv: 1407.5165].

[85] F. Brown, Multiple modular values for $\mathrm{SL}_{2}(Z)$, arXiv:1407.5167.

[86] C.R. Mafra and O. Schlotterer, Cohomology foundations of one-loop amplitudes in pure spinor superspace, arXiv:1408.3605 [INSPIRE].

[87] P.J. Cameron, Combinatorics. Topics, techniques, algorithms, Cambridge Univ. Pr., Cambridge U.K. (1994).

[88] J. Riordan, Introduction to combinatorial analysis, Dover Publications, U.S.A. (2002).

[89] R.P. Stanley, Enumerative combinatorics, second edition, Cambridge Univ. Pr., Cambridge U.K. (2012). 